\title{
The structure of the tame kernels of quadratic number fields (I)
}

\author{
by \\ H. R. QIN (Nanjing)
}

1. Introduction. We study the structure of the 2-Sylow subgroup of the tame kernel of a number field. Although many results exist in this direction, this subgroup is not yet well understood, even for quadratic number fields. Let $F$ be a number field with the ring of integers $O_{F}$. Let $K_{n} O_{F}$ denote the $n$th Quillen $K$-group of $O_{F}$. In particular, $K_{2} O_{F}$, the Milnor group of $O_{F}$, is isomorphic to the tame kernel of $F$. Recently, J. Rognes and C. Weibel [21] gave the explicit structure of $K_{n} O_{F}$ for some number fields. But it remains a big challenge, even for $n=2$ and $F$ a quadratic number field.

For a positive integer $n$, let $r_{2^{n}}=r_{2^{n}}\left(K_{2} O_{F}\right)=2^{n}$-rank of $K_{2} O_{F}$, i.e., the number of cyclic components of $K_{2} O_{F}$ whose order is divisible by $2^{n}$. To understand the 2-Sylow subgroup of $K_{2} O_{F}$, one needs to know $r_{2^{n}}$ for all $n$. We shall develop a method to treat $r_{4}$ when $F$ is a quadratic number field.

We shall now give a brief indication of the contents.

In Section 2, we recall some known results which will be used repeatedly in this paper, state some definitions, and derive some new results which give a method of determining $r_{4}$ for a quadratic field $F$.

In Sections 3 and 4, we use the results of the preceding section to study the 2-Sylow subgroups of the tame kernel of real and imaginary quadratic fields, respectively.

Let $F=\mathbb{Q}(\sqrt{d}), d \in \mathbb{Z}$ square-free, be a quadratic field. When $d$ has at most three odd prime divisors, we know $r_{4}$ in every case (see $\left.[18,19]\right)$.

Let $F=\mathbb{Q}(\sqrt{d})$ be a real quadratic field. We use $N F$ for the set of norms from $F$ over $\mathbb{Q}$. Let $d \in \mathbb{N}$ have prime factorization $d=2^{\sigma} l_{1} \cdots l_{n}$, where $\sigma=0$ or 1 .

2000 Mathematics Subject Classification: 11R11, 11R70, 19F15.

The author is supported by the National Distinguished Youth Science Foundation of China Grant and the 973 Grant. 
For $j=1,3,5,7$ we let $m_{j}$ denote the number of $l_{i}$ 's which are $\equiv j$ $(\bmod 8)$, and we call $2^{\sigma}\left(m_{1}, m_{3}, m_{5}, m_{7}\right)$ the type of $d$. It is proved by B. Brauckmann [1] that if $d$ has a positive divisor $\equiv 7(\bmod 8)$, then $r_{4} \geq 1$.

In Section 3, we give all real quadratic fields for which $r_{4} \geq 1$. More precisely, we have the following theorem:

Theorem A0. Let $F=\mathbb{Q}(\sqrt{d})$ be a real quadratic field. Then $r_{4}\left(K_{2} O_{F}\right)$ $\geq 1$ in the following cases:

(1) $d$ has a positive divisor $\equiv 7(\bmod 8)$;

(2) $d$ is odd and of type $\left(m_{1}, 0, m_{5}, 0\right)$ with $m_{5} \geq 2$ even;

(3) $d=2 d^{\prime}$ is even and $d^{\prime}$ is of type $\left(m_{1}, m_{3}, 0,0\right)$ with $m_{3} \geq 2$ even.

Note that (2) and (3) are equivalent to the following $\left(2^{\prime}\right)$ and $\left(3^{\prime}\right)$ respectively:

$\left(2^{\prime}\right) d \equiv 1(\bmod 8)$ with $-1 \in N F$ and $-2 \notin N F$;

$\left(3^{\prime}\right) d=2 d^{\prime}, d^{\prime} \equiv 1(\bmod 8)$ with $-1 \notin N F$ and $-2 \in N F$.

Let $p \equiv 1(\bmod 8)$ be a prime. It is known (Conner-Hurrelbrink [5]) that for $F=\mathbb{Q}(\sqrt{p})$ and also for $F=\mathbb{Q}(\sqrt{2 p})$ both sets of primes

$$
\begin{aligned}
& \left\{p \equiv 1(\bmod 8) \text { and } r_{4}\left(K_{2} O_{F}\right)=0\right\}, \\
& \left\{p \equiv 1(\bmod 8) \text { and } r_{4}\left(K_{2} O_{F}\right)=1\right\}
\end{aligned}
$$

have density $1 / 2$ in the set of all primes $p \equiv 1(\bmod 8)$.

The following theorem generalizes this phenomenon to more than one prime factor:

TheOREM A1. Let $n \geq 2$. Except for cases (1)-(3) listed above, there is a set of positive density consisting of square-free integers $d>0$ of given parity and given type $2^{\sigma}\left(m_{1}, m_{3}, m_{5}, m_{7}\right)$ such that for $F=\mathbb{Q}(\sqrt{d})$ the 4 rank of $\mathrm{K}_{2} \mathrm{O}_{F}$ is 0 , and there is a set of positive density of such d's with $r_{4}\left(K_{2} O_{F}\right) \geq 1$.

Therefore, in view of Wiles's result on the Birch-Tate conjecture, which predicts that $\left|K_{2} O_{F}\right|=\left|w_{2}(F) \zeta_{F}(-1)\right|$ for all totally real number fields, we have some information on the even part of $w_{2}(F) \zeta_{F}(-1)$. Here $w_{2}(F)$ is the order of the group of roots of unity in the algebraic closure of $F$ fixed by the square of the usual Galois action, and $\zeta_{F}(\cdot)$ is the Dedekind zeta function of $F$.

Theorems A0 and A1 follow from Theorem 3.1.

As a consequence of our results, we obtain necessary and sufficient conditions, involving only some Legendre symbols, for the 2-Sylow subgroup of $K_{2} O_{F}$ to be elementary abelian, i.e., $r_{4}=0$. This turns out to be the same as to give necessary and sufficient conditions for $r_{4}>0$. 
The same has also been done for imaginary quadratic fields in Section 4 . In fact, we have:

TheOREM B0. Let $F=\mathbb{Q}(\sqrt{-d}), d \in \mathbb{N}$ square-free, be an imaginary quadratic field. If $d \equiv 1(\bmod 8)$ with $d \neq 1$, then $r_{4} \geq 1$.

Unfortunately, for $F$ imaginary the analogue of Theorem A1 is not valid in general. But we have

Theorem B1. Let $F=\mathbb{Q}(\sqrt{-d}), d \in \mathbb{N}$ square-free, be an imaginary quadratic field. If $d \not \equiv 1(\bmod 8)$ has at least four odd prime divisors, then there is a set of positive density consisting of square-free integers $d^{\prime}>0$ with the same parity and the same type as $d$, such that for $F=\mathbb{Q}\left(\sqrt{-d^{\prime}}\right)$ the 4-rank of $\mathrm{K}_{2} \mathrm{O}_{F}$ is 0 ; and there exist infinitely many such d's with $r_{4}\left(K_{2} O_{F}\right)>0$. With some exceptional cases, the same is true for $d$ having at most three odd prime divisors.

Theorems B0 and B1 are consequences of Theorem 4.1, Corollary 4.1 and Remarks 1-3.

Again, as in real quadratic cases, we give necessary and sufficient conditions for the 2-Sylow subgroup of $K_{2} O_{F}$ to be elementary abelian.

Let us recall that in the imaginary quadratic case, a version of Lichtenbaum's conjecture reads as follows:

$$
\frac{3|D|^{3 / 2}}{\pi^{2} R_{2}(F)} \zeta_{F}(2)=\left|K_{2} O_{F}\right| .
$$

Here $D$ is the discriminant of $F=\mathbb{Q}(\sqrt{-d})$ and $R_{2}(F)$ a twisted version of the $m$ th Borel regulator.

2. Preliminaries. Let $d \neq 0$ be an integer. We will use the following notation:

$$
S(d)= \begin{cases}\{ \pm 1, \pm 2\} & \text { if } d>0 \\ \{1,2\} & \text { if } d<0\end{cases}
$$

For an abelian group $A, A_{2}$ will denote the 2-Sylow subgroup of $A$, and ${ }_{2} A=\left\{x \in A \mid x^{2}=1\right\}$. Let $F$ be a number field. Denote by $\Omega$ the set of all places of $F$.

For a finite place $\wp \in \Omega$, we use $\tau_{\wp}$ for the tame symbol at $\wp$. For any integer $n$, put $\nabla^{n}=\left\{\alpha \in K_{2} O_{F} \mid \alpha=\beta^{n}\right.$ for some $\left.\beta \in K_{2} O_{F}\right\}$.

Lemma 2.1 (Browkin and Schinzel [3]). Let $F=\mathbb{Q}(\sqrt{d}), d \in \mathbb{Z}$ squarefree. Then ${ }_{2} \mathrm{~K}_{2} \mathrm{O}_{F}$ is generated by

$$
\{-1, m\}, \quad m \mid d,
$$

together with

$$
\left\{-1, u_{i}+\sqrt{d}\right\}
$$


if $\{-1, \pm 2\} \cap N F \neq \emptyset$, where $u_{i} \in \mathbb{Z}$ is such that $u_{i}^{2}-d=c_{i} w_{i}^{2}$ for some $w_{i} \in \mathbb{Z}$ and $c_{i} \in\{-1, \pm 2\} \cap N F$.

LemMa $2.2([18,19])$. Let $F=\mathbb{Q}(\sqrt{d}), d \in \mathbb{Z}$ square-free. Suppose that $m \mid d(m>0$ if $d>0$, but $m$ also takes on negative values if $d<0)$ and write $d=u^{2}-2 w^{2}$ with $u, w \in \mathbb{Z}(u>0$ if $d>0)$ if $2 \in N F$. Then $\{-1, m\} \in \nabla^{2}$ if and only if one can find an $\varepsilon \in S(d)$ such that

(i) $\left(\frac{d / m}{p}\right)=\left(\frac{\varepsilon}{p}\right)$ for every odd prime $p \mid m$;

(ii) $\left(\frac{m}{p}\right)=\left(\frac{\varepsilon}{p}\right)$ for every odd prime $p \mid \frac{d}{m}$;

and $\{-1, m(u+\sqrt{d})\} \in \nabla^{2}$ if and only if one can find $a \delta \in S(d)$ such that

(iii) $\left(\frac{d / m}{p}\right)=\left(\frac{\delta(u+w)}{p}\right)$ for every odd prime $p \mid m$;

(iv) $\left(\frac{m}{p}\right)=\left(\frac{\delta(u+w)}{p}\right)$ for every odd prime $p \mid \frac{d}{m}$.

LEMmA 2.3. Let $F=\mathbb{Q}(\sqrt{d})$ be a quadratic field.

(i) Suppose $m \mid d$. Assume that $m>0$ if $d>0$, and $m \equiv 1(\bmod 4)$ if $d \equiv 1(\bmod 8)$. Then there is a prime $p \equiv 1(\bmod 4)$ such that

$$
\eta p m Z^{2}=X^{2}+d Y^{2}
$$

is solvable for $\eta=1$ or 2 . Moreover:

If $d \equiv 1(\bmod 2)$, then $\left(\frac{d}{p}\right)=1 ;$ furthermore, $\eta=2$ if $d \equiv 5(\bmod 8)$ and $m \equiv 3(\bmod 4)$, and $\eta=1$, otherwise.

For $d=2 d^{\prime}$, we have $\eta=1$, and

- if $m \equiv 1(\bmod 8)$, then $\left(\frac{d^{\prime}}{p}\right)=1$;

- if $m \equiv 5(\bmod 8)$, then $\left(\frac{d^{\prime}}{p}\right)=-1$;

- if $m \equiv 3(\bmod 8)$ and $d^{\prime} \equiv 1(\bmod 4)$ then $\left(\frac{d^{\prime}}{p}\right)=1$;

- if $m \equiv 3(\bmod 8)$ and $d^{\prime} \equiv 3(\bmod 4)$ then $\left(\frac{d^{\prime}}{p}\right)=-1$;

- if $m \equiv 7(\bmod 8)$ and $d^{\prime} \equiv 1(\bmod 4)$ then $\left(\frac{d^{\prime}}{p}\right)=-1$;

- if $m \equiv 7(\bmod 8)$ and $d^{\prime} \equiv 3(\bmod 4)$ then $\left(\frac{d^{\prime}}{p}\right)=1$.

(ii) Suppose $2 \in N F, d=u^{2}-2 w^{2}$, where $u, w \in \mathbb{Z}$ and $m \mid d$. Assume that $m u>0$ if $d>0$ and $m(u+w) \equiv 1(\bmod 4)$ if $d \equiv 1(\bmod 8)$. Then there is a prime $p \equiv 1(\bmod 4)$ such that

$$
p m(u+w) Z^{2}=X^{2}+d Y^{2}
$$

is solvable (the solvability of $p m(u+w) Z^{2}=X^{2}+d Y^{2}$ is equivalent to that of $\left.2 p m(u+w) Z^{2}=X^{2}+d Y^{2}\right)$. Moreover,

- if $d \equiv 1(\bmod 2)$, then $\left(\frac{d}{p}\right)=1$. 
The proof of this lemma can be found in [20]. We note that the statement of Lemma 2.3 is a more explicit version of Lemma 3.4 in [20].

Lemma 2.4. Let $F=\mathbb{Q}(\sqrt{d}), d \in \mathbb{Z}$ square-free. Suppose that $m \mid d$.

(i) $\{-1, m\} \in \nabla^{2}$ if and only if $\varepsilon p Z^{2}=X^{2}-d Y^{2}$ is solvable for some $\varepsilon \in S(d)$, where $p \equiv 1(\bmod 4)$ is a prime such that $\eta p m Z^{2}=X^{2}+d Y^{2}$ is solvable for $\eta=1$ or 2 .

(ii) If $2 \in N F$, then $\{-1, m(u+\sqrt{d})\} \in \nabla^{2}$ if and only if $\delta p Z^{2}=$ $X^{2}-d Y^{2}$ is solvable for some $\delta \in S(d)$, where $p \equiv 1(\bmod 4)$ is a prime such that $p m(u+w) Z^{2}=X^{2}+d Y^{2}$ is solvable.

Proof. (i) Let $X_{0}, Y_{0}, Z_{0} \in \mathbb{Z}$ with $\left(X_{0}, Y_{0}\right)=\left(X_{0}, Z_{0}\right)=\left(Y_{0}, Z_{0}\right)=1$ be a solution of $\eta p m Z^{2}=X^{2}+d Y^{2}$. It follows from $p \equiv 1(\bmod 4)$ that $p=a^{2}+b^{2}$ for some $a, b \in \mathbb{Z}$. Let $p O_{F}=\wp_{1} \wp_{2}$ and put

$$
\gamma=\left\{\frac{X_{0}}{\sqrt{d} Y_{0}}, \frac{X_{0}^{2}+d Y_{0}^{2}}{X_{0}^{2}}\right\}\left\{\frac{b}{a}, \frac{p}{b^{2}}\right\} .
$$

Then $\gamma^{2}=\{-1, m\}$. Computing the tame symbols at every finite (prime) place, we see that

$$
\tau_{\wp} \gamma= \begin{cases}-1 & \text { if } \wp=\wp_{1}, \\ 1 & \text { otherwise (including } \left.\wp=\wp_{2}\right) .\end{cases}
$$

Now the result follows from Lemma 3.1 of [17] and Lemma 3.1 of [18].

For the proof of (ii), see [18].

Consider the equation $\varepsilon p Z^{2}=X^{2}-d Y^{2}$, where $p \equiv 1(\bmod 4)$ is a prime such that $\eta p m Z^{2}=X^{2}+d Y^{2}$ (resp., $p m(u+w) Z^{2}=X^{2}+d Y^{2}$ ) is solvable for $\eta=1$ or 2 . Let $d=2^{\sigma} l_{1} \cdots l_{n}$ be the prime factorization, where $\sigma=0$ or 1 . Let $\delta_{i}=\left(\frac{\varepsilon p}{l_{i}}\right)$ for $1 \leq i \leq n$, where $\varepsilon \in S(d)$. We obtain a vector $v(p, \varepsilon)=\left(\delta_{1}, \ldots, \delta_{n}\right)$. The Legendre Theorem on the solvability of the quadratic homogeneous Diophantine equation $a X^{2}+b Y^{2}+c Z^{2}=0$ (see [9] or [13]) tells us that the above equation has a non-trivial solution if and only if all components of the vector $v(p, \varepsilon)$ are 1 . This implies the following:

LEMma 2.5. With the above notation, we have $\{-1, m\} \in \nabla^{2}$ or $\{-1$, $m(u+\sqrt{d})\} \in \nabla^{2}$ if and only if $v(p, \varepsilon)=(1, \ldots, 1)$ for some $\varepsilon \in S(d)$.

Definition 2.1. Let $F=\mathbb{Q}(\sqrt{d}), d \in \mathbb{Z}$, be a quadratic field. We know that ${ }_{2} K_{2} O_{F} \cap\left(K_{2} F\right)^{2}$ is finitely generated. A set $S=\left\{m_{1}, \ldots, m_{k}\right\}$ is called a system of $\nabla$-representatives of $F$ if $\left\{-1, m_{1}\right\}, \ldots,\left\{-1, m_{k}\right\}$ generate ${ }_{2} K_{2} O_{F} \cap\left(K_{2} F\right)^{2}$ and $m_{1}, \ldots, m_{k}$ are multiplicatively independent $\bmod \left(F^{* 2} \cup 2 F^{* 2}\right)$.

Remark 2.1. 1. Let $F=\mathbb{Q}(\sqrt{d})$ be a real quadratic field. Suppose that $m \mid d$. Recall from $[17,19]$ that $\{-1, m\} \in{ }_{2} K_{2} O_{F} \cap\left(K_{2} F\right)^{2}$ if and only if $m>0$ whenever $d \not \equiv 1(\bmod 8)$, and if and only if $m>0$ and $m \equiv 1(\bmod 4)$ 
whenever $d \equiv 1(\bmod 8)$. Moreover, $\{-1, m(u+\sqrt{d})\} \in{ }_{2} K_{2} O_{F} \cap\left(K_{2} F\right)^{2}$ if and only if $m>0$ whenever $d \not \equiv 1(\bmod 8)$, and if and only if $m>0$ and $m(u+w) \equiv 1(\bmod 4)$ whenever $d \equiv 1(\bmod 8)$.

2. Let $F=\mathbb{Q}(\sqrt{d})$ be an imaginary quadratic field. Suppose that $m \mid d$. Recall from [18] that if $d \not \equiv 1(\bmod 8)$, then $\{-1, m\} \in{ }_{2} K_{2} O_{F} \cap\left(K_{2} F\right)^{2}$; if $d \equiv 1(\bmod 8)$ then $\{-1, m\} \in{ }_{2} K_{2} O_{F} \cap\left(K_{2} F\right)^{2}$ if and only if $m \equiv 1$ $(\bmod 4)$. Moreover, $\{-1, m(u+\sqrt{d})\} \in{ }_{2} K_{2} O_{F} \cap\left(K_{2} F\right)^{2}$ if and only if $m>0$ whenever $d \not \equiv 1(\bmod 8)$, and if and only if $m(u+w) \equiv 1(\bmod 4)$ whenever $d \equiv 1(\bmod 8)$.

Let $n_{i} \mid d$ with $1 \leq i \leq t$. Assume $\eta_{i} p_{i} n_{i} Z^{2}=X^{2}+d Y^{2}\left(\right.$ or $\eta_{i} p_{i} n_{i}(u+w) Z^{2}$ $\left.=X^{2}+d Y^{2}\right)$ are solvable for primes $p_{i} \equiv 1(\bmod 4)$ and $\eta_{i}=1$ or 2 $(1 \leq i \leq t)$. Note that for any integers $X_{1}, Y_{1}, X_{2}, Y_{2}$, we have the identity

$$
\left(X_{1}^{2}+d Y_{1}^{2}\right)\left(X_{2}^{2}+d Y_{2}^{2}\right)=\left(X_{1} X_{2}-d Y_{1} Y_{2}\right)^{2}+d\left(X_{1} Y_{1}+X_{2} Y_{2}\right)^{2} .
$$

Therefore, the assumption implies that $\prod_{i=1}^{t} p_{i} \eta_{i} n_{i} Z^{2}=X^{2}+d Y^{2}$ (or $\left.\prod_{i=1}^{t} p_{i} \eta_{i} n_{i}(u+w)^{t} Z^{2}=X^{2}+d Y^{2}\right)$ is solvable.

Similarly, suppose that $n_{i} \mid d$ with $1 \leq i \leq t$. Assume $\eta_{i} p_{i} n_{i} Z^{2}=X^{2}+$ $d Y^{2}\left(1 \leq i \leq t_{1}\right)$ and $\eta_{i} p_{i} n_{i}(u+w) Z^{2}=X^{2}+d Y^{2}\left(t_{1}+1 \leq i \leq t\right)$ are solvable for primes $p_{i} \equiv 1(\bmod 4)$ and $\eta_{i}=1$ or $2(1 \leq i \leq t)$. Then $\prod_{i=1}^{t} p_{i} \eta_{i} n_{i}(u+w)^{t-t_{1}} Z^{2}=X^{2}+d Y^{2}$ is solvable.

Let $p \equiv \prod_{i=1}^{t} p_{i}(\bmod 4 d)$ be any prime. Then $p \prod_{i=1}^{t} \eta_{i} n_{i} Z^{2}=X^{2}+d Y^{2}$ (or $p \prod_{i=1}^{t} \eta_{i} n_{i}(u+w)^{t} Z^{2}=X^{2}+d Y^{2}$, or $p \prod_{i=1}^{t} \eta_{i} n_{i}(u+w)^{t-t_{1}} Z^{2}=$ $\left.X^{2}+d Y^{2}\right)$ is solvable. Set

$$
\delta_{j}=\left(\frac{\prod_{i=1}^{t} \eta_{i} \cdot p}{l_{j}}\right)
$$

and for $1 \leq i \leq t$ and $1 \leq j \leq n$, let

$$
\delta_{i, j}=\left(\frac{\eta_{i} p_{i}}{l_{j}}\right) .
$$

We observe that $\left(\delta_{1}, \ldots, \delta_{n}\right)=\left(\prod_{i=1}^{t} \delta_{i, 1}, \ldots, \prod_{i=1}^{t} \delta_{i, n}\right)$. When $2 \in N F$, all $\eta_{i}$ can be chosen to be 1 .

Keeping the same notation, we have

Lemma 2.6. $\left\{-1, n_{1} \cdots n_{t}\right\} \in \nabla^{2}$ if and only if

$$
v(p, \varepsilon)=\left(\left(\frac{\varepsilon}{l_{1}}\right) \prod_{i=1}^{t} \delta_{i, 1}, \ldots,\left(\frac{\varepsilon}{l_{n}}\right) \prod_{i=1}^{t} \delta_{i, n}\right)=(1, \ldots, 1)
$$

for some $\varepsilon \in S(d)$.

The above discussion leads to

Definition 2.2. Let $|d|=2^{\sigma} l_{1} \cdots l_{n}$. Let $S=\left\{m_{1}, \ldots, m_{k}\right\}$ be a system of $\nabla$-representatives of $F=\mathbb{Q}(\sqrt{d})$, and suppose that the equations 
$\eta_{i} p_{i} m_{i} Z^{2}=X^{2}+d Y^{2}$ (or $\eta_{i} p_{i} m_{i}(u+w) Z^{2}=X^{2}+d Y^{2}$ ) are solvable for primes $p_{i} \equiv 1(\bmod 4)$ and $\eta_{i} \in\{1,2\}$. Let $E=\left(\varepsilon_{1}, \ldots, \varepsilon_{k}\right) \in S(d)^{k}$. The sign matrix of $d$ with respect to $S=\left\{m_{1}, \ldots, m_{k}\right\}$ and $E=\left(\varepsilon_{1}, \ldots, \varepsilon_{k}\right)$ is the $k \times n$ matrix (with entries in $\{-1,1\}$ )

$$
M(d, S, E)=\left[\left(\frac{\varepsilon_{i} \eta_{i} p_{i}}{l_{j}}\right)\right] .
$$

As a particular case, taking $E=(1, \ldots, 1)$, we obtain the sign matrix

$$
M(d, S)=\left[\left(\frac{\eta_{i} p_{i}}{l_{j}}\right)\right],
$$

where

$$
\left(\frac{\eta_{i} p_{i}}{l_{j}}\right)= \begin{cases}\left(\frac{m_{i}}{l_{j}}\right)\left(\operatorname{resp} .,\left(\frac{(u+w) m_{i}}{l_{j}}\right)\right) & \text { if } l_{j} \nmid m_{i}, \\ \left(\frac{d / m_{i}}{l_{j}}\right)\left(\operatorname{resp} .,\left(\frac{(u+w) d / m_{i}}{l_{j}}\right)\right) & \text { if } l_{j} \mid m_{i}\end{cases}
$$

which we call the sign matrix with respect to the set $S$ of $\nabla$-representatives.

Sometimes we simply write $M(d)$ for $M(d, S, E)$ or $M(d, S)$ if we do not need to emphasize $S$ and $E$. For any given type $T$, let $d(T)$ denote the set of all positive integers of type $T$. In symbols, $d(T)=\{d \mid d \in \mathbb{N}$ of type $T\}$. If $d \in d(T)$ is not fixed, we also use $M(T)$ to denote $M(d)$, which is convenient since in most cases below we have to find a $d$ of a given type with a sign matrix as described.

For the exact size of a sign matrix, we have

LEMma 2.7. Let d be a square-free integer with $n$ odd prime divisors and let $M(d)$ be a sign matrix of size $k \times n$. Then:

- For real quadratic fields,

$$
k= \begin{cases}n & \text { if either }(\mathrm{a}) p \equiv 1(\bmod 8) \text { for every odd prime } p \mid d \text { and } \\ & u+w \equiv 1(\bmod 4), \text { or }(\mathrm{b}) d \not \equiv 1(\bmod 8) \text { and } 2 \in N F ; \\ n-1 & \text { if either }(\mathrm{a}) d \equiv 1(\bmod 8), 2 \notin N F \text { and } p \equiv 1(\bmod 4) \text { for } \\ & \text { every prime } p \mid d, \text { or }(\mathrm{b}) d \equiv 1(\bmod 8), 2 \in N F \text { but either } \\ & u+w \equiv 3(\bmod 4) \text { or } d \text { has a positive divisor } \equiv 3(\bmod 4), \\ & \text { or }(\mathrm{c}) d \not \equiv 1(\bmod 8) \text { and } 2 \notin N F ; \\ n-2 & \text { if } d \equiv 1(\bmod 8), 2 \notin N F \text { and } d \text { has a positive divisor } \\ & \equiv 3(\bmod 4) .\end{cases}
$$


- For imaginary quadratic fields,

$$
k= \begin{cases}n & \text { if } d \equiv 1(\bmod 8) \text { and } 2 \in N F \\ n-1 & \text { if } d \equiv 1(\bmod 8) \text { and } 2 \notin N F \\ n+1 & \text { if } d \not \equiv 1(\bmod 8) \text { and } 2 \in N F \\ n & \text { if } d \not \equiv 1(\bmod 8) \text { and } 2 \notin N F\end{cases}
$$

Proof. Let $F=\mathbb{Q}(\sqrt{d})$ be a real quadratic field. Let $d=2^{\sigma} l_{1} \cdots l_{n}$ be the prime factorization, where $\sigma=0$ or 1 . Assume that $d \not \equiv 1(\bmod 8)$. Then by Remarks 2.1, we may choose $\left\{l_{1}, \ldots, l_{n-1}\right\}$ if $2 \notin N F$, and $\left\{l_{1}, \ldots, l_{n-1}, u+\right.$ $\sqrt{d}\}$ if $2 \in N F$, as a system of $\nabla$-representatives of $F$. If $d \equiv 1(\bmod 8)$, we write $d=l_{1} \cdots l_{m} \cdot l_{m+1} \cdots l_{n}$, where $l_{i} \equiv 3(\bmod 4)(1 \leq i \leq m)$, and $l_{j} \equiv 1$ $(\bmod 4)(m+1 \leq j \leq n)$. By Remarks 2.1 again, we can choose a system of $\nabla$-representatives of $F$ to be $\left\{l_{1} l_{2}, l_{1} l_{3}, \ldots, l_{1} l_{m}, l_{m+1}, \ldots, l_{n-1}\right\}$ if $2 \notin N F$; $\left\{l_{1} l_{2}, l_{1} l_{3}, \ldots, l_{1} l_{m}, l_{m+1}, \ldots, l_{n-1}, u+\sqrt{d}\right\}$ if $2 \in N F$ and $u+w \equiv 1(\bmod 4)$; and $\left\{l_{1} l_{2}, l_{1} l_{3}, \ldots, l_{1} l_{m}, l_{m+1}, \ldots, l_{n-1}, l_{1}(u+\sqrt{d})\right\}$ if $2 \in N F$ and $u+w \equiv 3$ $(\bmod 4)$.

Note that for an imaginary quadratic field $F=\mathbb{Q}(\sqrt{d}),-1$ can be chosen as an element in a system of $\nabla$-representatives if $d \not \equiv 1(\bmod 8)$. If $d \equiv 1$ $(\bmod 8)$ has a prime divisor $p \equiv 3(\bmod 4)$, then $-p \equiv 1(\bmod 4)$. Similarly, we can assume that $u+w \equiv 1(\bmod 4)$ if $d \equiv 1(\bmod 8)$. So the result follows and the lemma is proved.

REMARK 2.2. In [8], J. Hurrelbrink and M. Kolster introduced a kind of sign matrix to compute $r_{4}\left(K_{2} O_{F}\right)$ via local Hilbert symbols.

Let $M(T)=\left[\delta_{i, j}\right]$ be a sign matrix of $d$. The following operations are called elementary operations:

(I) Multiplying the $i$ th row of $M(T)$ by the $j$ th row. (This corresponds to replacing $m_{i}$ by $m_{i} m_{j}$ in the set of $\nabla$-representatives of $F$ - see the discussion after Remarks 2.1.)

(II) Interchanging the $i$ th and $j$ th rows. (This corresponds to interchanging $m_{i}$ and $m_{j}$.)

(II') Interchanging the $i$ th and $j$ th columns. (This corresponds to interchanging $l_{i}$ and $l_{j}$.)

(III) Multiplying the $i$ th row by a vector $\left(\delta_{1}, \ldots, \delta_{n}\right)$ where $\delta_{j}=\left(\frac{\varepsilon}{l_{j}}\right)$ for some $\varepsilon \in S(d)$. (This corresponds to changing the $i$ th entry in the set $E=\left(\varepsilon_{1}, \ldots, \varepsilon_{k}\right)$.)

However, one should be careful when applying elementary operations $(\mathrm{II})$ if $l_{i} \not \equiv l_{j}(\bmod 8)$ since in elementary operations (III) one may have $\left(\frac{\varepsilon}{l_{i}}\right) \neq\left(\frac{\varepsilon}{l_{j}}\right)$. So, one has to remember the congruences of $l_{i}$ and $l_{j}(\bmod 8)$. Usually, we fix a suitable order of $\left\{l_{i}\right\}$. 
Assume that $A$ and $B$ are sign matrices. We say that $A$ is equivalent to $B$ and write $A \cong B$ if $A$ can be transformed into $B$ by a sequence of elementary operations. We call a row in a sign matrix totally positive if its entries are all 1.

For a quadratic field $F=\mathbb{Q}(\sqrt{d})$, if $d$ has exactly $n$ odd prime divisors $l_{1}, \ldots, l_{n}$, then for a system of $\nabla$-representatives of $F$, we have only finitely many different sign matrices, and at most $4^{n}$ if we fix the order of $l_{1}, \ldots, l_{n}$. Any two of them are equivalent. Clearly, if $\{-1, m\} \in \nabla^{2}$, then $m\left(\bmod F^{* 2} \cup 2 F^{* 2}\right)$ is the product of some elements in a system of $\nabla$-representatives of $F$. Multiplying the rows corresponding to these generators and by a suitable choice of $\varepsilon$, i.e., multiplying by $\left(\left(\frac{\varepsilon}{l_{1}}\right), \ldots,\left(\frac{\varepsilon}{l_{n}}\right)\right)$, we get a totally positive row. Conversely, if some elementary operations yield a totally positive row, then we have an element $\{-1, m\} \in{ }_{2} K_{2} O_{F} \cap\left(K_{2} F\right)^{2}$ such that $\{-1, m\} \in \nabla^{2}$.

REMARK 2.3 . We can see easily that a totally positive row can be obtained (if at all) by the application of elementary operations (I) and (II) and at most one elementary operation (III), carried out as the last step of the process. See Lemma 2.6.

Lemma 2.8. Let $F=\mathbb{Q}(\sqrt{d})$ be a quadratic field, where $d$ is square-free and has $n$ odd prime divisors. Consider sign matrices of size $k \times n$, viewed as matrices over $\mathbb{Z} / 2 \mathbb{Z}$. Then $r_{4}\left(K_{2} O_{F}\right)$ coincides with the maximum of $k-r$ if $F$ is real, and $k-r-1$ if $F$ is imaginary, where $r$ runs through the values of ranks of all sign matrices of $F$.

The following is another property of a sign matrix.

Lemma 2.9. Let $d \in \mathbb{Z}$ be square-free, and let $M(d, S, E)$ be the sign matrix of $d$ with respect to $S=\left\{m_{1}, \ldots, m_{k}\right\}$ and $E=\left(\varepsilon_{1}, \ldots, \varepsilon_{k}\right)$.

Suppose $d$ is odd. Then the product of all entries in the ith row of $M(d, S, E)$ is:

- $\left(\frac{\varepsilon_{i}}{|d|}\right)$ if $d \not \equiv 5(\bmod 8)$ or $m_{i} \not \equiv 3(\bmod 4)$;

- $-\left(\frac{\varepsilon_{i}}{|d|}\right)$ if $d \equiv 5(\bmod 8)$ and $m_{i} \equiv 3(\bmod 4)$.

Suppose $d=2 d^{\prime}$ is even and $m_{i} \mid d$. Then the product of all entries in the ith row of $M(d, S, E)$ is $\left(\frac{\varepsilon_{i}}{\left|d^{\prime}\right|}\right)\left(\frac{d^{\prime}}{p}\right)$, where $\left(\frac{d^{\prime}}{p}\right)$ has been given explicitly in Lemma 2.3 (by taking $m=m_{i}$ ).

Proof. Let $d=2^{\sigma} l_{1} \cdots l_{n}$ be the prime factorization, where $\sigma=0$ or 1 . Then

$$
M(d, S, E)=\left[\delta_{i, j}\right]=\left[\left(\frac{\varepsilon_{i} \eta_{i} p_{i}}{l_{j}}\right)\right] .
$$


So,

$$
\prod_{j=1}^{n} \delta_{i, j}=\prod_{j=1}^{n}\left(\frac{\varepsilon_{i} \eta_{i} p_{i}}{l_{j}}\right)= \begin{cases}\left(\frac{\varepsilon_{i} \eta_{i} p_{i}}{|d|}\right) & \text { if } d \text { is odd } \\ \left(\frac{\varepsilon_{i} \eta_{i} p_{i}}{\left|d^{\prime}\right|}\right) & \text { if } d=2 d^{\prime} \text { is even. }\end{cases}
$$

If $d$ is odd, then $\left(\frac{p_{i}}{|d|}\right)=\left(\frac{d}{p_{i}}\right)$ since $p_{i} \equiv 1(\bmod 4)$. By Lemma 2.3, we know that $\left(\frac{d}{p_{i}}\right)=1$, moreover, if $d \not \equiv 5(\bmod 8)$ or $m_{i} \not \equiv 3(\bmod 4)$ then $\eta_{i}=1$, so

$$
\left(\frac{\varepsilon_{i} \eta_{i} p_{i}}{|d|}\right)=\left(\frac{\varepsilon_{i}}{|d|}\right)
$$

and if $d \equiv 5(\bmod 8)$ and $m_{i} \equiv 3(\bmod 4)$, then $\eta_{i}=2$, so

$$
\left(\frac{\varepsilon_{i} \eta_{i} p_{i}}{|d|}\right)=-\left(\frac{\varepsilon_{i}}{|d|}\right) \text {. }
$$

Similarly, if $d=2 d^{\prime}$ is even, then $\eta_{i}=1$ by Lemma 2.3 , so

$$
\left(\frac{\varepsilon_{i} \eta_{i} p_{i}}{\left|d^{\prime}\right|}\right)=\left(\frac{\varepsilon_{i}}{\left|d^{\prime}\right|}\right)\left(\frac{d^{\prime}}{p_{i}}\right)
$$

and the lemma is proved.

Lemma 2.10. Let $n \geq 2$ be an integer. Assume that for $1 \leq i<j \leq n$ and $1 \leq k \leq n$ we are given $\varepsilon_{i j} \in\{ \pm 1\}$ and odd integers $t_{k}$. Then there are infinitely many integers $d$ such that $d$ has exactly $n$ odd prime divisors, say, $l_{1}, \ldots, l_{n}$, with $\left(\frac{l_{i}}{l_{j}}\right)=\varepsilon_{i j}$ and $l_{k} \equiv t_{k}(\bmod 8)$ for all $1 \leq i<j \leq n, 1 \leq k \leq n$. Moreover, for any fixed $n-1$ primes, say, $l_{1}, \ldots, l_{n-1}$, and any given odd integer $t$, the set $\left\{l \mid\left(\frac{l_{i}}{l}\right)=\varepsilon_{\text {in }}, 1 \leq i \leq n-1\right\}$ has a positive density as a subset of the set of all primes $\equiv t(\bmod 8)$.

Proof. We argue by induction on $n$. Since there are infinitely many primes in an arithmetical progression, which is needed throughout the proof, we may choose $l_{1} \equiv t_{1}(\bmod 8)$ to be a prime. Let $c_{2}$ be an integer such that $c_{2} \equiv t_{2}(\bmod 8)$ and $\left(\frac{c_{2}}{l_{1}}\right)=\varepsilon_{12}$ if $t_{1}$ or $t_{2} \equiv 1(\bmod 4)$, while $\left(\frac{c_{2}}{l_{1}}\right)=-\varepsilon_{12}$ if $t_{1} \equiv t_{2} \equiv 3(\bmod 8)$. Then we can find a prime $l_{2}$ from the arithmetical progression $8 l_{1} k+c_{2}$.

Suppose that we have primes $l_{1}, \ldots, l_{n-1}$ with $\left(\frac{l_{i}}{l_{j}}\right)=\varepsilon_{i j}$ and $l_{i} \equiv t_{i}$ $(\bmod 8)$ for $1 \leq i<j \leq n-1$. Then $l_{n}$ can be chosen from the arithmetical progression $8 l_{1} \cdots l_{n-1} k+c_{n}$, where $c_{n} \equiv t_{n}(\bmod 8)$ and $\left(\frac{c_{n}}{l_{j}}\right)=\varepsilon_{j n}(1 \leq$ $j \leq n-1)$. The Dirichlet density theorem implies that $\left\{l \mid\left(\frac{l_{i}}{l}\right)=\varepsilon_{i n}, 1 \leq\right.$ $i \leq n-1\}$ has a positive density. The lemma is proved. 


\section{Real quadratic fields}

Definition 3.1. For any given type $2^{\sigma}\left(m_{1}, m_{3}, m_{5}, m_{7}\right)$ and any given odd primes $p_{1}, \ldots, p_{t}$, we say that the set $\left\{p_{1}, \ldots, p_{t}\right\}$ satisfies $\left(m_{1}, m_{3}, m_{5}, m_{7}\right)$ if the product $p_{1} \cdots p_{t}$ is of type $\left(n_{1}, n_{3}, n_{5}, n_{7}\right)$ with $n_{i} \leq m_{i}$ for $i=1,3,5,7$. In this paper, except for the case where we say that a prime $p$ has type $T$, we always assume that $\sum\left(m_{i}-n_{i}\right)=1$.

Theorem 3.1. Let $F=\mathbb{Q}(\sqrt{d}), d \in \mathbb{N}$ square-free, be a real quadratic field. Then $r_{4}\left(K_{2} O_{F}\right) \geq 1$ in the following cases:

(i) $d$ has a positive divisor $d^{\prime} \equiv 7(\bmod 8)$;

(ii) $d$ is odd and of type $\left(m_{1}, 0, m_{5}, 0\right)$ with $m_{5} \geq 2$ even;

(iii) $d$ is even and of type $2\left(m_{1}, m_{3}, 0,0\right)$ with $m_{3} \geq 2$ even.

For each of the types

(iv) $\left(m_{1}, 0, m_{5}, 0\right)$ with $m_{5}$ odd and $m_{1}+m_{5} \geq 2$;

(v) $2\left(m_{1}, 0, m_{5}, 0\right)$ with $m_{5}>0$ and $m_{1}+m_{5} \geq 3$;

(vi) $\left(m_{1}, m_{3}, 0,0\right)$ with $m_{3}>0$ and $m_{1}+m_{3} \geq 3$;

(vii) $2\left(m_{1}, m_{3}, 0,0\right)$ with $m_{3}$ odd and $m_{1}+m_{3} \geq 2$;

(viii) $\left(m_{1}, 0,0,0\right)$;

(ix) $2\left(m_{1}, 0,0,0\right)$,

the following is true:

If $T=2^{\delta}\left(m_{1}, m_{3}, m_{5}, m_{7}\right)$ is one of these types and if $\Lambda=\left\{p_{1}, \ldots, p_{t}\right\}$ is any set of odd primes satisfying $\left(m_{1}, m_{3}, m_{5}, m_{7}\right)$, let

$$
P(T, \Lambda)=\left\{\text { p prime } \mid d=2^{\delta} p p_{1} \cdots p_{t} \text { is of type } T\right\} .
$$

Then the sets of primes

$$
P_{0}(T, \Lambda)=\left\{p \in P(T, \Lambda) \mid r_{4}\left(K_{2} O_{\mathbb{Q}(\sqrt{d})}\right)=0\right\}
$$

and

$$
P_{1}(T, \Lambda)=\left\{p \in P(T, \Lambda) \mid r_{4}\left(K_{2} O_{\mathbb{Q}(\sqrt{d})}\right) \geq 1\right\}
$$

have positive density.

REMARK 3.1. Some cases for which we know everything about $r_{2}$ and $r_{4}$ are not included in the theorem. Let $F=\mathbb{Q}(\sqrt{d})$ be a real quadratic field. If $d=p$ or $2 p$ with $p$ a prime, then for $p \equiv \pm 3(\bmod 8)$, it is easy to see that $r_{4}\left(K_{2} O_{F}\right)=0$. For $p \equiv 7(\bmod 8)$, we always have $r_{4}\left(K_{2} O_{F}\right)=1$ (see, e.g., [1]). For $p \equiv 1(\bmod 8)$, see [5]. If $d$ has 2 or 3 odd prime divisors, then $r_{4}\left(K_{2} O_{F}\right)$ is listed completely in [19]. See also [16] for densities of 4-ranks of $K_{2} O_{F}$.

Proof. (i) See [1], also [8] and [20]. We will give another proof after showing (ii). 
(ii) We see from Lemma 2.9 that in this case for any sign matrix $M(d, S, E)$ of $d$ with respect to $S=\left\{m_{1}, \ldots, m_{k}\right\}$ and $E=\left(\varepsilon_{1}, \ldots, \varepsilon_{k}\right)$, the product of all entries in any row of $M(d, S, E)$ is 1 since $d \equiv 1(\bmod 8)$, which follows from the assumption that $m_{5}$ is even.

First we assume that $m_{1}=0$, i.e., $d$ has no prime divisor $p \equiv 1(\bmod 8)$. Keeping the notation as before, we suppose that $M(d)=\left[\delta_{i, j}\right]$ is the sign matrix with respect to $\left\{p_{1}, \ldots, p_{m_{5}-1}\right\}$. If $\delta_{i, j}=1$ for all $i, j$, then $r_{4}=$ $m_{5}-1 \geq 1$, so we are done. Assume now that $\delta_{i, j}=-1$ for some $i, j$. Performing some elementary operations if necessary, we may assume that $\delta_{1,1}=-1$. If $\delta_{i, 1}=-1$ for some $i>1$, then multiplying the $i$ th row by the first row, we see that

$$
M(d)=\left(\begin{array}{cccc}
-1 & \delta_{1,2} & \ldots & \delta_{1, m_{5}} \\
1 & & & \\
\vdots & & N & \\
1 & & &
\end{array}\right),
$$

where $N$ is an $\left(m_{5}-2\right) \times\left(m_{5}-1\right)$ sign matrix. If every entry of $N$ is 1 , then we have $r_{4} \geq 1$ again; if not, then as before we may assume that $N$ has $(1,1)$ entry -1 . We may assume that $\delta_{1,2}=1$ (otherwise multiply the first row of $M(d)$ by the second).

For $N$, we perform elementary operations just as for $M(d)$ to get

$$
M(d) \cong\left(\begin{array}{ccc}
-1 & 1 & \ldots \\
1 & -1 & \ldots \\
\vdots & \vdots & \\
1 & 1 & \ldots
\end{array}\right) .
$$

Here in the first and second columns, $M(d)$ has -1 in the $(1,1)$ and $(2,2)$ positions and 1 elsewhere. We repeat this process. If some row of an equivalent form of $M(d)$ is all 1's, then $r_{4} \geq 1$. Otherwise, we end up with

$$
M(d) \cong\left(\begin{array}{cccc}
-1 & & \ldots & * \\
& -1 & \ldots & * \\
\vdots & \vdots & & \\
& & \ldots & *
\end{array}\right),
$$

where the $(i, i)$ entries are -1 , and all other entries are 1 , except in the last column: every $*$ must be -1 , since the product of all entries in any row is 1 . Multiplying the first row by the remaining $m_{5}-2$ rows, we see that

$$
M(d) \cong\left(\begin{array}{cccc}
-1 & -1 & \ldots & -1 \\
& \ddots & & \vdots \\
& & -1 & -1
\end{array}\right),
$$

where the first row has -1 's everywhere, and the fact that the last entry is -1 follows from the assumption that $m_{5} \equiv 0(\bmod 2)$. 
The above argument works also for $m_{1} \geq 1$. One sees that the only case in which we might have $r_{4}=0$ is

$$
M(d) \cong\left(\begin{array}{cccc}
-1 & & & -1 \\
& \ddots & & \vdots \\
& & -1 & -1
\end{array}\right) .
$$

Here is another way to see this. Let $M$ be a sign matrix and let $M^{\prime}$ be the $\left(m_{5}+m_{1}-1\right) \times m_{5}$ matrix consisting of the first $m_{5}$ columns of $M$. Suppose that $M^{\prime}$ has at least $m_{1}$ totally positive rows; without loss of generality, the last $m_{1}$ rows of $M^{\prime}$ are totally positive. Then

$$
M=\left(\begin{array}{ccccc} 
\pm 1 & \ldots & & \ldots & \pm 1 \\
\vdots & & & & \vdots \\
\pm 1 & \ldots & & \ldots & \pm 1 \\
1 & \ldots & 1 & & \\
\vdots & & \vdots & N & \\
1 & \ldots & 1 & &
\end{array}\right)
$$

where $N$ is an $m_{1} \times m_{1}$ matrix. (Remember, however, that we do not want to interchange columns corresponding to the primes $p_{i}$ and columns corresponding to the primes $l_{j}$.) Then the point here is that the column-rank of $N$ is at most $m_{1}-1$, because of the condition that the product of the entries in any row is 1 , and hence the row-rank of the square matrix over $\mathbb{Z} / 2 \mathbb{Z}$ is also at most $m_{1}-1$. So a suitable sequence of row operations on the last $m_{1}$ rows of $M$ will give a totally positive row.

Thus, if at any stage we obtain a sign matrix $M$ for which $M^{\prime}$ has at least $m_{1}$ totally positive rows, we are done. If not, we end up with a sign matrix as above which is equivalent to $M$.

As usual, we may assume that the first $m_{5}$ columns correspond to $p_{1}, \ldots, p_{m_{5}}$. Multiplying the first row by rows 2 to $m_{5}$, we see that $M(d)$ is equivalent to a sign matrix whose first row is

$$
(\overbrace{-1, \ldots,-1}^{m_{5}}, \overbrace{1, \ldots, 1}^{m_{1}})=:\left(-1^{\cdots m_{5}}, 1^{\cdots m_{1}}\right) .
$$

In both cases ( $m_{1}=0$ or not), applying an elementary operation (III) by taking $\varepsilon=2$, we obtain a new equivalent form of $M(d)$, whose first row is all 1's. Thus we have shown that $r_{4} \geq 1$.

We use the above approach to prove (i):

CASE $1: m_{3}+m_{5} \neq 0$. Note that for any two primes $p, q$, if both $p, q \not \equiv 1$ $(\bmod 8)$ and $p \not \equiv q(\bmod 8)$, then for any $\delta_{1}, \delta_{2} \in\{ \pm 1\}$, there exists $\varepsilon \in$ $\{ \pm 1, \pm 2\}$ such that

$$
\left(\frac{\varepsilon}{p}\right) \delta_{1}=\left(\frac{\varepsilon}{q}\right) \delta_{2}=1 .
$$


CASE $2: m_{3}=m_{5}=0, m_{7} \neq 0$. If $d \equiv 1(\bmod 8)$ is of type $\left(m_{1}, 0,0, m_{7}\right)$, then the product of all entries in a row of any $A \cong M(d)$ is always 1 . In this case, $M(d)$ is of size $\left(m_{1}+m_{7}-1\right) \times\left(m_{1}+m_{7}\right)$. If $d \not \equiv 1(\bmod 8)$ is of type $\left(m_{1}, 0,0, m_{7}\right)$ or $d$ is even of type $2\left(m_{1}, 0,0, m_{7}\right)$, then $M(d)$ is of size $\left(m_{1}+m_{7}\right) \times\left(m_{1}+m_{7}\right)$. Applying an elementary operation (III), we can assume that one column in $M(d)$, corresponding to a fixed prime $p \equiv 7$ $(\bmod 8)$, is totally positive.

In both cases, by elementary operations (I) and (II), we can transform $M(d)$ into a sign matrix which has at least one totally positive row.

(iii) is the same as (ii).

Now we prove that when the type $T=2^{\sigma}\left(m_{1}, m_{3}, m_{5}, 0\right)$ belongs to one of cases (iv)-(ix) of the theorem, then for any $\Lambda$ satisfying $\left(m_{1}, m_{3}, m_{5}, 0\right)$ the sets $P_{0}(T, \Lambda)$ and $P_{1}(T, \Lambda)$ have positive density.

For each of the given types $T=2^{\sigma}\left(m_{1}, m_{3}, m_{5}, 0\right)$, we describe a matrix $M_{0}(T)$ with \pm 1 entries for which no combination of elementary operations will produce a totally positive row (see Step 1 of case (iv) below).

Now, given a set $\Lambda=\left\{p_{1}, \ldots, p_{n-1}\right\}$ of odd primes satisfying $\left(m_{1}, m_{3}\right.$, $\left.m_{5}, 0\right)$ and a choice, $S$, of $\nabla$-representatives for type $T$, one gets a sign matrix with respect to $S$ (described below in case (iv)). One can show that for a suitable choice of $S$, it is possible to find $p \in P(T, \Lambda)$ for which the sign matrix can be transformed by elementary operations to $M_{0}(T)$ (see Step 2 of case (iv)).

Lemma 2.10 guarantees that the set of such $p$ 's has positive density. Lemma 2.8, together with the stated property of $M_{0}(T)$, guarantees that the set of such $p$ 's is contained in $P_{0}(T, \Lambda)$ (see Step 3 of case (iv)).

We will explain the structure of the argument by treating the case (iv) in detail. For each of the remaining cases, we will give $M_{0}(T)$, but we will omit the verification (apart from some illustrations in cases (v) and (vii)) since it is a routine matter and similar to case (iv).

So let us consider case (iv): $T=\left(m_{1}, 0, m_{5}, 0\right), m_{5} \equiv 1(\bmod 2)$ and $m_{5}+m_{1} \geq 2$.

STEP 1. Put

$$
M_{0}(T)=\left(\begin{array}{cccc}
-1 & & & -1 \\
& \ddots & & \vdots \\
& & -1 & -1
\end{array}\right) .
$$

Here $M_{0}(T)$ is an $\left(m_{5}+m_{1}-1\right) \times\left(m_{5}+m_{1}\right)$ sign matrix, whose $(i, i)$ and $\left(i, m_{5}+m_{1}\right)$ entries are -1 and all other entries are 1 .

For a given type $T$ and $d \in d(T)$, if $M(d)$ is a sign matrix of $d$, we recall that a necessary and sufficient condition for $r_{4}\left(K_{2} O_{\mathbb{Q}(\sqrt{d})}\right) \geq 1$ is that $M(d)$ is equivalent to a matrix having all entries 1 on some rows. It is easy to see 
that we have to make all signs below $p_{1}, \ldots, p_{m_{5}}$ equal if we want to get a totally positive row.

Following Remark 2.3, we apply elementary operations (I) and (II) to $M_{0}(T)$. Multiplying the first row by rows 2 to $m_{5}$, which is the only way to make all signs below $p_{1}, \ldots, p_{m_{5}}$ equal, we see that the first row will be

$$
\left(-1^{\cdots m_{5}}, 1^{\cdots m_{1}-1},-1\right)
$$

where the last component is -1 because $m_{5} \equiv 1(\bmod 2)$. Since for any choice of $\varepsilon$, there is no way to change the last component -1 into 1 , we obtain $r_{4}=0$.

SteP 2. Given a set $\Lambda=\left\{p_{1}, \ldots, p_{n-1}\right\}$ of odd primes satisfying $T=$ $\left(m_{1}, 0, m_{5}, 0\right)$ (remember that $\left.n=m_{1}+m_{5}\right)$ with $m_{5}$ odd, we are going to show that one can find $p \in P(T, \Lambda)$ for which the sign matrix of $p_{1} \cdots p_{n-1} \cdot p$ can be transformed by elementary operations to $M_{0}(T)$.

We choose $S=\Lambda=\left\{p_{1}, \ldots, p_{n-1}\right\}$ as a system of $\nabla$-representatives of $F=\mathbb{Q}\left(\sqrt{p_{1} \cdots p_{n-1} \cdot p}\right)$. Let $2 \leq i \leq n$ be any integer. We let the $i$ th column correspond to $p$, and the $j$ th column correspond to $p_{j}$ if $j<i$, and to $p_{j+1}$ if $j \geq i$. Then the sign matrix $M(T)$ of $p_{1} \cdots p_{n-1} \cdot p$ is

$$
\left(\begin{array}{cccccccc}
x_{1,1} & a_{1,2} & \ldots & a_{1, i-1} & y_{1, i} & a_{1, i+1} & \ldots & a_{1, n} \\
a_{2,1} & x_{2,2} & \ldots & a_{2, i-1} & y_{2, i} & a_{2, i+1} & \ldots & a_{2, n} \\
\vdots & \vdots & \ddots & \vdots & \vdots & \vdots & & \vdots \\
a_{i-1,1} & a_{i-1,2} & \ldots & x_{i-1, i-1} & y_{i-1, i} & a_{i-1, i+1} & \ldots & a_{i-1, n} \\
a_{i, 1} & a_{i, 2} & \ldots & a_{i, i-1} & y_{i, i} & x_{i, i+1} & \ldots & a_{i, n} \\
\vdots & \vdots & \ddots & \vdots & \vdots & \vdots & \ddots & \vdots \\
a_{n-1,1} & a_{n-1,2} & \ldots & a_{n-1, i-1} & y_{n-1, i} & a_{n-1, i+1} & \ldots & x_{n-1, n}
\end{array}\right),
$$

where

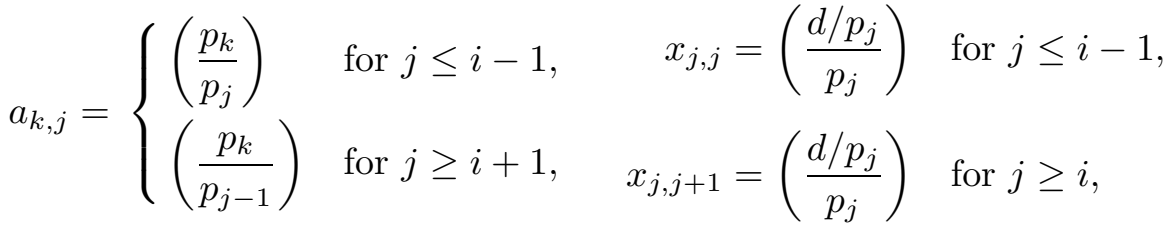

$$
\begin{aligned}
& y_{k, i}=\left(\frac{p_{k}}{p}\right) \quad \text { for any } k \text {. }
\end{aligned}
$$

Note that $x_{j, j}(j \leq i-1)$ and $x_{j, j+1}(j \geq i)$ may take any value \pm 1 .

Apply elementary operations (I) and (II) to $M(T)$ to convert it to $M_{0}(T)$ :

We begin by setting $x_{1,1}=-1$. Apply elementary row operations to obtain 1 below $x_{1,1}$. Then set $x_{2,2}=-a_{1,2}\left(a_{1,2}=a_{2,1}\right)$. The $(2,2)$ entry is now -1 by some elementary row operations, and we obtain 1 below $x_{2,2}$. The method can be extended to $x_{i-1, i-1}$, and so the $(i-1, i-1)$ entry is -1 
and all elements below $x_{i-1, i-1}$ are 1 after giving $x_{i-1, i-1}$ a suitable value and applying some elementary row operations.

We continue our process by assigning $x_{i, i+1}$ a suitable value $( \pm 1)$ such that the $(i+1, i+1)$ entry is now -1 . Then the elements below the $(i+1, i+1)$ entry can be made 1 . Repetition of the process leads to a sign matrix (equivalent to $M(T)$ ) with 1 below any $x_{j, j+1}$.

Now, apply elementary row operations to eliminate all -1 above $x_{j, j}$ and $x_{j, j+1}$.

Note that when the value of $x_{j, j}\left(x_{j, j+1}\right)$ is given, the value of $y_{j, i}$ is obtained by the rule that the product of all entries in any row is 1 .

Thus, with a suitable choice of the values of $x_{j, j}$ or $x_{j, j+1}$ and by elementary operations (I) and (II), we obtain a sign matrix

$$
M(T) \cong\left(\begin{array}{cccccccc}
-1 & 1 & \ldots & 1 & -1 & 1 & \ldots & 1 \\
1 & -1 & \ldots & 1 & -1 & 1 & \ldots & 1 \\
\vdots & \vdots & \ddots & \vdots & \vdots & \vdots & & \vdots \\
1 & 1 & \ldots & -1 & -1 & 1 & \ldots & 1 \\
1 & 1 & \ldots & 1 & -1 & -1 & \ldots & 1 \\
\vdots & \vdots & \ddots & \vdots & \vdots & \vdots & \ddots & \vdots \\
1 & 1 & \ldots & 1 & -1 & 1 & \ldots & -1
\end{array}\right)
$$

where again all elements in the $i$ th column are -1 because the product of all entries in any row is 1.

Multiplying rows 1 to $n-2$ by the last row, we obtain a new equivalent form of $M(T)$ :

$$
M(T) \cong\left(\begin{array}{cccccccc}
-1 & 1 & \ldots & 1 & 1 & 1 & \ldots & -1 \\
1 & -1 & \ldots & 1 & 1 & 1 & \ldots & -1 \\
\vdots & \vdots & \ddots & \vdots & \vdots & \vdots & & \vdots \\
1 & 1 & \ldots & -1 & 1 & 1 & \ldots & -1 \\
1 & 1 & \ldots & 1 & 1 & -1 & \ldots & -1 \\
\vdots & \vdots & \ddots & \vdots & \vdots & \vdots & \ddots & \vdots \\
1 & 1 & \ldots & 1 & -1 & 1 & \ldots & -1
\end{array}\right) .
$$

Let $N$ be the submatrix of the above matrix consisting of rows $i$ to $n-2$. By interchanging the $n$th and $(n-1)$ th rows, which can be realized by a series of elementary row operations, we see that the above matrix is $\cong M_{0}(T)$.

STEP 3. Finally we show that the density of $P_{0}(T, \Lambda)$ is positive.

In fact, taking

$$
i= \begin{cases}m_{5} & \text { if } p \equiv 5(\bmod 8), \\ m_{5}+1 & \text { if } p \equiv 1(\bmod 8)\end{cases}
$$


one sees from the discussion above that $p \in P_{0}(T, \Lambda)$ if and only if for each $1 \leq j \leq n-1,\left(\frac{p_{j}}{p}\right)$ takes a suitable value from $\{ \pm 1\}$. So the result follows from Lemma 2.10.

The claim that $P_{1}(T, \Lambda)$ has a positive density will be proved after we present $M_{0}(T)$ for cases (v)-(ix).

One can check that each $M_{0}(T)$ given below is really a sign matrix for some $d \in d(T)$, and that $r_{4}=0$.

In case (iv), it is an immediate consequence of Lemma 2.10 that there are (infinitely many) $d \in d(T)$ such that $M(d) \cong M_{0}(T)$. In each of the cases below, we can easily see that $M_{0}(T)$ is equivalent to a sign matrix with entries 1 everywhere except for the principal diagonal and the last column. The reason that we first present $M_{0}(T)$ rather than its equivalent form is that we want to convince the reader that $M_{0}(T)$ is a sign matrix for some $d \in d(T)$ according to Lemma 2.10, although it is more convenient for us to use that equivalent form to verify that $r_{4}=0$ and that the density of $P_{0}(T, \Lambda)$ is positive.

So for each $M_{0}(T)$, by Lemma 2.10, we can choose $d \in d(T)$ with prime factorization $d=2^{\sigma} p_{1} \cdots p_{m_{t}} l_{1} \cdots l_{m_{1}}$, where $t=3$ or 5 , i.e., $p_{i} \equiv 5$ or 3 $(\bmod 8), l_{j} \equiv 1(\bmod 8)\left(1 \leq i \leq m_{t}, 1 \leq j \leq m_{1}\right)$ and $\sigma=0$ or 1 such that $M(d)=M_{0}(T)$. Except for case (vi) with $m_{3} \equiv 0(\bmod 2)$, we always choose $S=\left\{p_{1}, \ldots, p_{m_{t}}, l_{1}, \ldots, l_{m_{1}-1}\right\}$ as a system of $\nabla$-representatives. In fact, one may begin by letting $p_{1} \equiv t(\bmod 8)\left(\right.$ or $\left.l_{1} \equiv 1(\bmod 8)\right)$ be any prime and then pick up the remaining primes such that the Legendre symbols concerning them are as described in $M_{0}(T)$.

We make the following assumption:

In a sign matrix, we arrange the columns so that the first $m_{t}$ columns correspond to all primes $\equiv t(\bmod 8)$ and the last $m_{1}$ columns correspond to primes $\equiv 1(\bmod 8)$, where $t=3$ or 5 .

Now Lemma 2.10 implies that there are infinitely many $d \in d(T)$ such that $M(d)=M_{0}(T)$. Of course, this does not mean that $P_{0}(T, \Lambda)$ has a positive density, which requires a proof as in case (iv).

(v) $T=2\left(m_{1}, 0, m_{5}, 0\right)$ :

If $m_{1} \leq 1$, let

$$
\begin{aligned}
& M_{0}(T)=\left(\begin{array}{ccccc}
1 & & & & -1 \\
& -1 & & & 1 \\
& & \ddots & & \vdots \\
& & & -1 & 1
\end{array}\right) \\
& \left(\delta_{1, m_{5}+m_{1}}=-1, \delta_{i, i}=-1,2 \leq i \leq m_{5}+m_{1}-1\right) ;
\end{aligned}
$$


if $m_{1} \geq 2$, let

$$
\begin{aligned}
& M_{0}(T)=\left(\begin{array}{ccccccc}
1 & & & & & & \\
& -1 & & & & & 1 \\
& & \ddots & & & & \vdots \\
& & & -1 & & & 1 \\
& & & -1 & & -1 \\
& & & & \ddots & & \vdots \\
& & & & & -1 & -1
\end{array}\right) \\
& \left(\delta_{1, m_{5}+m_{1}}=-1, \delta_{i, i}=\delta_{j, m_{5}+m_{1}}=-1,\right. \\
& \left.2 \leq i \leq m_{5}+m_{1}-1, m_{5}+1 \leq j \leq m_{5}+m_{1}-1\right) \text {. }
\end{aligned}
$$

In both cases, multiplying rows 2 to $m_{5}$, we get

$$
M_{0}(T) \cong\left(\begin{array}{ccccc}
1 & & & & -1 \\
& -1 & & & -1 \\
& & \ddots & & \vdots \\
& & & -1 & -1
\end{array}\right) .
$$

Given a set of $\Lambda=\left\{p_{1}, \ldots, p_{n-1}\right\}$ odd primes satisfying $T=2\left(m_{1}, 0, m_{5}, 0\right)$ $\left(n=m_{1}+m_{5}\right)$, we suppose that $p_{1} \equiv \cdots \equiv p_{k} \equiv 5(\bmod 8)$ and $p_{k+1} \equiv$ $\cdots \equiv p_{n-1} \equiv 1(\bmod 8)$. Choose $S=\left\{p_{1}, p_{1} p_{2}, \ldots, p_{1} p_{k}, p_{k+1}, \ldots, p_{n-1}\right\}$ as a system of $\nabla$-representatives of $F=\mathbb{Q}\left(\sqrt{p_{1} \cdots p_{n-1} \cdot p}\right)$. Let $2 \leq i \leq n$ be any integer. We let the $i$ th column correspond to $p$, and the $j$ th column correspond to $p_{j}$ if $j<i$, and to $p_{j+1}$ if $j \geq i$. Then we obtain the sign matrix $M(T)$. Setting $x_{1,1}=1$ and applying an elementary operation (III) by taking $E=\left(\varepsilon_{1}, \ldots, \varepsilon_{n-1}\right)$, where $\varepsilon_{j}=1$ if $j=1$ or $a_{j, 1}=1$, and $\varepsilon_{j}=2$ if $a_{j, 1}=-1$, we see that all elements in the first column are 1 . Then the same discussion as in (iv) gives the desired result.

(vi) $T=\left(m_{1}, m_{3}, 0,0\right)$ :

(a) If $m_{3} \equiv 1(\bmod 2)$, let

$$
\begin{aligned}
& M_{0}(T)=\left(\begin{array}{ccccccc}
-1 & & & & & -1 \\
\vdots & \ddots & & & & \vdots \\
-1 & \ldots & -1 & & & -1 \\
& & & -1 & & -1 \\
& & & & \ddots & & \vdots \\
& & & & & -1 & -1
\end{array}\right) \\
& \left(\delta_{i, j}=-1, \delta_{i, m_{3}+m_{1}}=(-1)^{i}, 1 \leq j \leq i \leq m_{3} ;\right. \\
& \left.\delta_{i, i}=\delta_{i, m_{3}+m_{1}}=-1, m_{3}+1 \leq i \leq m_{3}+m_{1}-1\right) \text {. }
\end{aligned}
$$


We have

$$
\begin{aligned}
& M_{0}(T) \cong\left(\begin{array}{cccc}
-1 & & & -1 \\
& \ddots & & \vdots \\
& & -1 & -1
\end{array}\right) \\
& \left(\delta_{i, i}=-1, \delta_{i, m_{3}+m_{1}}=-1,1 \leq j \leq i \leq m_{3}+m_{1}\right) \text {. }
\end{aligned}
$$

(b) If $m_{3} \equiv 0(\bmod 2)$, let

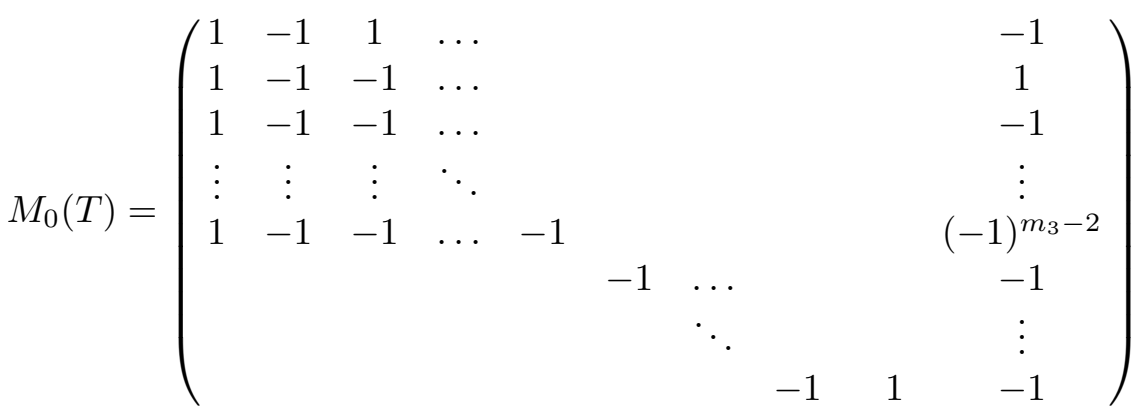

$$
\begin{aligned}
& \left(\delta_{i, j}=-1, \delta_{i, m_{3}+m_{1}}=(-1)^{i}, 1 \leq i \leq m_{3}-1,2 \leq j \leq i+1\right. \text {; } \\
& \left.\delta_{i, i}=\delta_{i, m_{3}+m_{1}}=-1, m_{3} \leq i \leq m_{3}+m_{1}-2\right) \text {. }
\end{aligned}
$$

Then

$$
\begin{gathered}
M_{0}(T) \cong\left(\begin{array}{ccccccc}
1 & -1 & 1 & \ldots & 1 & 1 & -1 \\
1 & 1 & -1 & \ldots & 1 & 1 & 1 \\
1 & 1 & 1 & \ldots & 1 & 1 & -1 \\
\vdots & \vdots & \vdots & \ddots & \vdots & \vdots & \vdots \\
1 & 1 & 1 & \ldots & 1 & -1 & -1
\end{array}\right) \\
\left(\delta_{i, i+1}=-1, \delta_{i, m_{3}+m_{1}}=-1,1 \leq i \leq m_{3}+m_{1}-2\right)
\end{gathered}
$$

and $r_{4}=0$.

We have a little difference here. Note that for any $x \in \mathbb{Z}$ with $x \equiv 3$ $(\bmod 4), x$ is not the sum of two squares in $F=\mathbb{Q}(\sqrt{d})$, since $d \equiv 1(\bmod 8)$.

We choose $d \in d(T)$ with prime factorization $d=p_{1} \cdots p_{m_{3}} l_{1} \cdots l_{m_{1}}$, where $p_{i} \equiv 3(\bmod 8)\left(1 \leq i \leq m_{3}\right)$ and $l_{j} \equiv 1(\bmod 8)\left(1 \leq j \leq m_{1}\right)$. We choose $\left\{p_{1} p_{2}, p_{1} p_{3}, \ldots, p_{1} p_{m_{3}}, l_{1}, \ldots, l_{m_{1}-1}\right\}$ as a system of $\nabla$-representatives. Suppose that $q_{i} \equiv 1(\bmod 4)\left(1 \leq i \leq m_{3}+m_{1}-2\right)$ are primes such that either $q_{i} p_{1} p_{i+1} Z^{2}=X^{2}+d Y^{2}$ if $1 \leq i \leq m_{3}-2$, or $q_{m_{3}-2+i} l_{i} Z^{2}=X^{2}+d Y^{2}$ if $1 \leq i \leq m_{1}$, have non-trivial solutions in $\mathbb{Z}$. Letting $p_{1} \equiv 3(\bmod 8)$ be any prime, we choose $p_{j}$ with $\left(\frac{p_{j}}{p_{1}}\right)=1$ for all $2 \leq j \leq m_{3}$ such that $\left(\frac{p_{j}}{p_{i}}\right)(i \neq j, i \neq 1)$ are as described in $M_{0}(T)$.

(vii) $2\left(m_{1}, m_{3}, 0,0\right)$ with $m_{3}$ odd and $m_{1}+m_{3} \geq 2$ : 
If $m_{1} \leq 1$, let

$$
\begin{aligned}
& M_{0}(T)=\left(\begin{array}{ccccc}
1 & 1 & 1 & \ldots & -1 \\
-1 & 1 & 1 & \ldots & 1 \\
-1 & -1 & 1 & \ldots & -1 \\
\vdots & \vdots & \vdots & \ddots & \vdots \\
-1 & -1 & -1 & \ldots & (-1)^{m_{3}+m_{1}-1}
\end{array}\right) \\
& \left(\delta_{i, j}=-1, \delta_{k, m_{3}+m_{1}}=(-1)^{k}, 1 \leq j<i \leq m_{3}+m_{1}-1,1 \leq k \leq m_{3}+m_{1}-1\right) \text {; } \\
& \text { if } m_{1} \geq 2 \text {, let } \\
& \begin{array}{c}
M_{0}(T)=\left(\begin{array}{ccccccc}
1 & 1 & 1 & \ldots & & -1 \\
-1 & 1 & 1 & \ldots & & 1 \\
-1 & -1 & 1 & \ldots & & -1 \\
\vdots & \vdots & \vdots & \ddots & & \vdots \\
-1 & -1 & -1 & \ldots & 1 & \ldots & -1\left((-1)^{m_{3}}\right) \\
& & & & -1 & -1 \\
& & & & & & -1
\end{array}\right) \\
\left(\delta_{i, j}=-1, \delta_{k, m_{3}+m_{1}}=(-1)^{k}, 1 \leq j<i \leq m_{3}, 1 \leq k \leq m_{3} ;\right. \\
\left.\delta_{i, i}=\delta_{i, m_{3}+m_{1}}=-1, m_{3}+1 \leq i \leq m_{3}+m_{1}-1\right) .
\end{array}
\end{aligned}
$$

By elementary row operations (including interchanging the first and $(n-1)$ th rows), we see that in both cases

$$
\begin{gathered}
M_{0}(T) \cong\left(\begin{array}{cccccc}
-1 & 1 & 1 & \ldots & 1 & -1 \\
1 & -1 & 1 & \ldots & 1 & -1 \\
\vdots & \vdots & \vdots & & \vdots & \vdots \\
1 & 1 & 1 & \ldots & -1 & -1 \\
1 & 1 & 1 & \ldots & 1 & -1
\end{array}\right) \\
\left(\delta_{1, m_{3}+m_{1}}=-1, \delta_{i+1, i}=-1,1 \leq i \leq m_{3}+m_{1}-1\right) .
\end{gathered}
$$

Given a set $\Lambda=\left\{p_{1}, \ldots, p_{n-1}\right\}$ of odd primes satisfying $T=2\left(m_{1}, m_{3}, 0,0\right)$ $\left(n=m_{1}+m_{3}\right)$, we suppose that $p_{1} \equiv \cdots \equiv p_{k} \equiv 3(\bmod 8)$ and $p_{k+1} \equiv$ $\cdots \equiv p_{n-1} \equiv 1(\bmod 8)$. Choose $S=\left\{p_{1} p_{2}, \ldots, p_{1} p_{k}, p_{k+1}, \ldots, p_{n-1}, p_{1}\right\}$ as a system of $\nabla$-representatives of $F=\mathbb{Q}\left(\sqrt{p_{1} \cdots p_{n-1} \cdot p}\right)$. Then repeat the same discussion as in (iv).

Observe that for $m_{1}=0$ and $m_{1}>0$, the $M_{0}(T)$ are essentially the same.

We now use Lemmas 2.2 and 2.10 to show that for cases (iv)-(vii) the density of $P_{1}(T, \Lambda)$ is positive.

It is sufficient to show that for any given type $T=2^{\delta}\left(m_{1}, m_{3}, m_{5}, 0\right)$, where $\delta=0$ or 1 , and any given set $\Lambda=\left\{p_{1}, \ldots, p_{n-1}\right\}$ of primes $(n=$ 
$\left.m_{1}+m_{3}+m_{5}\right)$ satisfying $\left(m_{1}, m_{3}, m_{5}, 0\right)$, there exist primes $p \in P(T, \Lambda)$ such that $\{-1, m\} \in \nabla^{2}$ for some divisor $m$ of $p_{1} \cdots p_{n-1} \cdot p$. Moreover, the condition for $\{-1, m\} \in \nabla^{2}$ will be $\left(\frac{p_{i}}{p}\right)=\varepsilon_{i}$ for $\varepsilon_{i}= \pm 1(1 \leq i \leq n-1)$, so Lemma 2.10 implies that the density of $P_{1}(T, \Lambda)$ is positive.

First of all, for any given type $T=2^{\delta}\left(m_{1}, m_{3}, m_{5}, 0\right)$, where $\delta=0$ or 1 , and any given primes $p_{1}, \ldots, p_{n-1}$, if $p_{1} \cdots p_{n-1}$ is of type $\left(m_{1}-1, m_{3}, m_{5}, 0\right)$, then the density of $P_{1}(T, \Lambda)$ is obviously positive. In fact, we can take primes $p \equiv 1(\bmod 8)$ such that $\left(\frac{p_{i}}{p}\right)=1$ for $1 \leq i \leq n-1$. Clearly, $\{-1, p\} \in \nabla^{2}$.

We now suppose that $p_{1} \cdots p_{n-1}$ is of type $\left(m_{1}, m_{3}-1,0,0\right)$ or $\left(m_{1}, 0\right.$, $\left.m_{5}-1,0\right)$ :

- $T=\left(m_{1}, 0, m_{5}, 0\right)$ : We choose $p \equiv 5(\bmod 8)$ such that $\left(\frac{p_{1}}{p}\right)=1$ for $1 \leq i \leq n-1$ and we have $\{-1, p\} \in \nabla^{2}$.

- $T=2\left(m_{1}, 0, m_{5}, 0\right), m_{5} \equiv 1(\bmod 2):$ We choose $p \equiv 5(\bmod 8)$ such that $\left(\frac{p_{i}}{p}\right)=-1$ for all $p_{i} \equiv 5(\bmod 8)$ and $\left(\frac{p_{i}}{p}\right)=1$ for all $p_{i} \equiv 1(\bmod 8)$ and taking $\varepsilon=2$ we have $\{-1, p\} \in \nabla^{2}$.

- $T=2\left(m_{1}, 0, m_{5}, 0\right), m_{5} \equiv 0(\bmod 2):$ Suppose that $p_{1} \equiv 5(\bmod 8)$. If $\left(\frac{p_{2} \cdots p_{n-1}}{p_{1}}\right)=1$, we choose $p \equiv 5(\bmod 8)$ such that $\left(\frac{p_{i}}{p}\right)=\left(\frac{p_{i}}{p_{1}}\right)$ for all $p_{i} \equiv 1$ $(\bmod 8)$ and $\left(\frac{p_{i}}{p}\right)=-\left(\frac{p_{i}}{p_{1}}\right)$ for all $p_{i} \equiv 5(\bmod 8)$ and taking $\varepsilon=2$ we have $\left\{-1, p p_{1}\right\} \in \nabla^{2}$. If $\left(\frac{p_{2} \cdots p_{n-1}}{p_{1}}\right)=-1$, we choose $p \equiv 5(\bmod 8)$ such that $\left(\frac{p_{i}}{p}\right)=\left(\frac{p_{i}}{p_{1}}\right)$ for $1 \leq i \leq n-1$ and taking $\varepsilon=1$ we have $\left\{-1, p p_{1}\right\} \in \nabla^{2}$.

- $T=\left(m_{1}, m_{3}, 0,0\right), m_{3} \equiv 1(\bmod 2):$ Suppose that $p_{1} \equiv 5(\bmod 8) . \mathrm{We}$ choose $p \equiv 3(\bmod 8)$ such that $\left(\frac{p}{p_{i}}\right)=1$ for $1 \leq i \leq n-1$ and taking $\varepsilon=1$ we have $\{-1, p\} \in \nabla^{2}$.

- $T=\left(m_{1}, m_{3}, 0,0\right), m_{3} \equiv 0(\bmod 2):$ Suppose that $p_{1} \equiv 3(\bmod 8)$. If $\left(\frac{p_{2} \cdots p_{n-1}}{p_{1}}\right)=1$, we choose $p \equiv 3(\bmod 8)$ such that $\left(\frac{p}{p_{i}}\right)=\left(\frac{p_{1}}{p_{i}}\right)$ for $2 \leq i \leq n-1$ and taking $\varepsilon=1$ we have $\left\{-1, p p_{1}\right\} \in \nabla^{2}$. If $\left(\frac{p_{2} \cdots p_{n-1}}{p_{1}}\right)=-1$, we choose $p \equiv 3(\bmod 8)$ such that $\left(\frac{p}{p_{i}}\right)\left(\frac{p_{1}}{p_{i}}\right)=\left(\frac{2}{p_{i}}\right)$ for $2 \leq i \leq n-1$ and taking $\varepsilon=2$ we have $\left\{-1, p p_{1}\right\} \in \nabla^{2}$.

- $T=2\left(m_{1}, m_{3}, 0,0\right), m_{3} \equiv 1(\bmod 2)$ : We choose $p \equiv 3(\bmod 8)$ such that $\left(\frac{p}{p_{i}}\right)=\left(\frac{2}{p_{i}}\right)$ for $1 \leq i \leq n-1$ and taking $\varepsilon=2$ we have $\{-1, p\} \in \nabla^{2}$.

For the cases $T=\left(m_{1}, 0,0,0\right)$ and $T=2\left(m_{1}, 0,0,0\right)$, we shall use some results from [5] to show that both $P_{0}(T, \Lambda)$ and $P_{1}(T, \Lambda)$ have positive density. In [16], R. Osburn used a similar technique to study the densities of 4-ranks of $\mathrm{K}_{2} \mathrm{O}_{F}$.

(viii) $T=\left(m_{1}, 0,0,0\right)$ : For any prime $p \equiv 1(\bmod 8)$, we have an expression $p=u^{2}-2 w^{2}$ with $u, w \in \mathbb{N}$, and $\left(\frac{u+w}{p}\right)=1$ if and only if $u+w \equiv 1$ $(\bmod 4)$ if and only if $p=x^{2}+32 y^{2}$ for some $x, y \in \mathbb{Z}$ (see [5]). Note that if $p \equiv q \equiv 1(\bmod 8)$ with $p=u_{1}^{2}-2 w_{1}^{2}$ and $q=u_{2}^{2}-2 w_{2}^{2}$, where 
$u_{1}, u_{2}, w_{1}, w_{2} \in \mathbb{N}$, then there are $u, w \in \mathbb{N}$ such that $p q=u^{2}-2 w^{2}$; moreover, $u+w \equiv\left(u_{1}+w_{1}\right)\left(u_{2}+w_{2}\right)(\bmod 4)$.

Let $N$ denote the normal closure of $\mathbb{Q}(\sqrt{1+\sqrt{2}})$. Then the Galois group $\operatorname{Gal}(N / \mathbb{Q})$ is the dihedral group of order 8 . For a prime $p \equiv 1(\bmod 8)$, we have $p=x^{2}+32 y^{2}$ for some $x, y \in \mathbb{N}$ if and only if $p$ splits completely in $N$ if and only if for any prime ideal $P \subset O_{E}$ with $P \mid p$, the Artin symbol $\left(\frac{N / \mathbb{Q}}{P}\right)$ is trivial.

For any given primes $p_{i} \equiv 1(\bmod 8), 1 \leq i \leq m_{1}-1$ (with prescribed values $\left.\left(\frac{p_{i}}{p_{j}}\right)\right)$, by suitable choice of a prime $p \equiv 1(\bmod 8)$, we may make the modified sign matrix $M^{\prime}(d)$ for $d=p_{1} \cdots p_{m_{1}-1} \cdot p$ equivalent to

$$
\left(\begin{array}{cccc}
-1 & & & -1 \\
& \ddots & & \vdots \\
& & -1 & -1
\end{array}\right),
$$

where -1 appears exactly in all $(i, i)$ entries and the last column. Here we have removed $u+\sqrt{d}$ from a system of $\nabla$-representatives, so $M^{\prime}(d)$ is not a sign matrix if $u+w \equiv 1(\bmod 4)\left(d=u^{2}-2 w^{2}\right)$. We know that if $d=p_{1} \cdots p_{m_{1}-1} \cdot p$ is chosen to correspond to $M^{\prime}(d)$, then for any positive divisor $m$ of $d,\{-1, m\} \notin \nabla^{2}$. On the other hand, a necessary and sufficient condition for $\{-1, m(u+\sqrt{d})\} \in \nabla^{2}$ is that the Diophantine equation $m(u+w) Z^{2}=X^{2}-d Y^{2}$ has a non-trivial solution in $\mathbb{Z}$. It follows immediately that if $\left(\frac{u+w}{d}\right)=-1$, then $\{-1, m(u+\sqrt{d})\} \notin \nabla^{2}$. So if $\left(\frac{u+w}{d}\right)=-1$ together with $M^{\prime}(d)$ being as above, then $r_{4}=0$.

Now applying the Chebotarev Density Theorem to the number field $E$ which is the compositum of $N$ and $\mathbb{Q}\left(\sqrt{p_{i}}\right), 1 \leq i \leq m_{1}-1$, we see that for any given primes $p_{i} \equiv 1(\bmod 8), 1 \leq i \leq m_{1}-1, P_{0}(T, \Lambda)$ has a positive density.

(ix) $2\left(m_{1}, 0,0,0\right)$ is similar. In fact, we take the same sign matrix as in the case $\left(m_{1}, 0,0,0\right)$. Analogously, we see that if $\left(\frac{u+w}{d / 2}\right)=-1\left(d=u^{2}-2 w^{2}\right)$, then for any divisor $m$ of $d,\{-1, m(u+\sqrt{d})\} \notin \nabla^{2}$. Note that $\left(\frac{u+w}{d / 2}\right)=-1$ if and only if $\left(\frac{-2}{u+w}\right)=-1$. On the other hand, if $d / 2=u^{\prime 2}-2{w^{\prime}}^{2} \equiv 1(\bmod 8)$, then $w^{\prime}$ is even and $d=\left(2 u^{\prime}+2 w^{\prime}\right)^{2}-2\left(u^{\prime}+2 w^{\prime}\right)^{2}$. Hence $\left(\frac{-2}{u+w}\right)=-1$ if and only if $\left(\frac{-2}{u^{\prime}}\right)=-1$. Observe that if $p \equiv q \equiv 1(\bmod 8)$ with $p=u_{1}^{2}-2 w_{1}^{2}$ and $q=u_{2}^{2}-2 w_{2}^{2}$, where $u_{1}, u_{2}, w_{1}, w_{2} \in \mathbb{N}$, then there are $U, W \in \mathbb{N}$ such that $p q=U^{2}-2 W^{2} ;$ moreover, $U \equiv u_{1} \cdot u_{2}(\bmod 8)$. For a prime $p \equiv 1(\bmod 8)$ with $p=u^{2}-2 w^{2}$ for some $u, w \in \mathbb{N}$, it follows from [5] that $\left(\frac{-2}{u}\right)=-1$ if and only if either $p \neq x^{2}+32 y^{2}$ for all $x, y \in \mathbb{N}$ and $p \equiv 1(\bmod 16)$, or $p=x^{2}+32 y^{2}$ for some $x, y \in \mathbb{N}$ and $p \equiv 9(\bmod 16)$. Let $E=\mathbb{Q}\left(\zeta_{16}\right)$. It is easy to see that $p \equiv 1(\bmod 16)$ if and only if $p$ splits completely in $E$ if and only if for any prime ideal $P \subset O_{E}$ with $P \mid p$, the Artin symbol $\left(\frac{E / \mathbb{Q}}{P}\right)$ 
is trivial. As in the case $T=\left(m_{1}, 0,0,0\right)$, applying the Chebotarev Density Theorem, we deduce again that $P_{0}(T, \Lambda)$ has a positive density.

This concludes the proof.

Corollary 3.1. Let $F=\mathbb{Q}(\sqrt{d})$ with $d \in d(T)$. Then in cases (iv)(ix), $\left(K_{2} O_{F}\right)_{2}$ is elementary abelian if and only if $M(d) \cong M_{0}(T)$.

By [26], we have

Corollary 3.2. Let $r_{2}$ be the 2-rank of $\mathrm{K}_{2} \mathrm{O}_{F}$. In cases (i)-(iii), we have $2^{r_{2}+1} \mid w_{2}(F) \zeta_{F}(-1)$. And in cases (iv)-(ix), $2^{r_{2}} \| w_{2}(F) \zeta_{F}(-1)$ if and only if $M(d) \cong M_{0}(T)$.

Remark 3.1. Let $T=(2,1,0,0)$. Suppose that

$$
\Lambda_{1}=\left\{p, q \text { primes } \mid p, q \equiv 3,1(\bmod 8),\left(\frac{p}{q}\right)=1\right\} .
$$

Then $P\left(T, \Lambda_{1}\right)=\{l$ prime $\mid p \cdot q \cdot l$ is of type $T\}=\{l$ prime $\mid l \equiv 1(\bmod 8)\}$, the set $P_{0}\left(T, \Lambda_{1}\right)=\left\{l \in P\left(T, \Lambda_{1}\right) \mid r_{4}\left(K_{2} O_{\mathbb{Q}(\sqrt{p q l})}\right)=0\right\}$ has density $3 / 4$, and $P_{1}\left(T, \Lambda_{1}\right)=\left\{l \in P\left(T, \Lambda_{1}\right) \mid r_{4}\left(K_{2} O_{\mathbb{Q}(\sqrt{p q l})}\right)=1\right\}$ has density $1 / 4$.

On the other hand, if we put

$$
\Lambda_{2}=\left\{p, q \text { primes } \mid p, q \equiv 3,1(\bmod 8),\left(\frac{p}{q}\right)=-1\right\},
$$

then $P\left(T, \Lambda_{2}\right)=\{l$ prime $\mid p \cdot q \cdot l$ is of type $T\}=\{l$ prime $\mid l \equiv 1(\bmod 8)\}$, the set $P_{0}\left(T, \Lambda_{1}\right)=\left\{l \in P\left(T, \Lambda_{1}\right) \mid r_{4}\left(K_{2} O_{\mathbb{Q}(\sqrt{p q l})}\right)=0\right\}$ has density $1 / 4$, and $P_{1}\left(T, \Lambda_{1}\right)=\left\{l \in P\left(T, \Lambda_{1}\right) \mid r_{4}\left(K_{2} O_{\mathbb{Q}(\sqrt{p q l})}\right)=1\right\}$ has density $3 / 4$.

This example shows that $P_{0}(T, \Lambda)$ and $P_{1}(T, \Lambda)$ depend on the Legendre symbol $\left(\frac{p}{q}\right)$.

So in general, given a type $T$ and a set $\Lambda$ of odd primes, the densities of $P_{0}(T, \Lambda)$ and $P_{1}(T, \Lambda)$ as subsets of $P(T, \Lambda)$ depend not only on $T$ and $\Lambda$, but also on the Legendre symbols concerning the primes in $\Lambda$.

So we may ask:

Question. What are the exact values of the densities of $P_{0}(T, \Lambda)$ and $P_{1}(T, \Lambda) ?$

4. Imaginary quadratic fields. For convenience, we introduce the following

Notation. Let $d \in \mathbb{N}$ be square-free of type $2^{\sigma}\left(m_{1}, m_{3}, m_{5}, m_{7}\right)$. Write $m$ for $m_{1}, n$ for $m_{3}, s$ for $m_{5}$ and $t$ for $m_{7}$. With this notation, $d$ or $d / 2$ equals $d_{1} \cdot d_{3} \cdot d_{5} \cdot d_{7}$, where $d_{1}=l_{1} \cdots l_{m}, d_{3}=p_{1} \cdots p_{n}, d_{5}=q_{1} \cdots q_{s}$ and $d_{7}=r_{1} \cdots r_{t}$ with $l_{i} \equiv 1(\bmod 8), p_{j} \equiv 3(\bmod 8), q_{k} \equiv 5(\bmod 8)$ and $r_{h} \equiv 7$ $(\bmod 8)$ are primes $(1 \leq i \leq m, 1 \leq j \leq n, 1 \leq k \leq s$ and $1 \leq h \leq t)$. 
For a given type $T=2^{\sigma}\left(m_{1}, m_{3}, m_{5}, m_{7}\right)$, where $\sigma=0$ or 1 , we say $T \equiv 1(\bmod 8)$ if $\sigma=0$ and $m_{3} \equiv m_{5} \equiv m_{7}(\bmod 2)$; otherwise, $T \not \equiv 1$ $(\bmod 8)$.

We introduce the following two kinds of sign matrices:

$$
D(k)=\left(\begin{array}{cccc}
-1 & & & -1 \\
& \ddots & & \vdots \\
& & -1 & -1
\end{array}\right),
$$

where $D(k)$ is of size $(k-1) \times k$ with -1 in the indicated places only, and

$$
\begin{aligned}
& E(k, l)=\left(\begin{array}{ccccccccc}
-1 & 1 & 1 & \ldots & & & & & -1 \\
-1 & -1 & 1 & \ldots & & & & 1 \\
-1 & -1 & -1 & \ldots & & & & -1 \\
\vdots & \vdots & \vdots & \ddots & & & & \vdots \\
-1 & -1 & -1 & \ldots & -1 & 1 & \ldots & (-1)^{k} \\
& & & & & -1 & \ldots & -1 \\
& & & & & & \ddots & & \vdots \\
& & & & & & & -1 & -1
\end{array}\right) \\
& \left(\delta_{i, j}=-1, \delta_{i, k+l}=(-1)^{i}, 1 \leq j \leq i \leq k ;\right. \\
& \left.\delta_{i, i}=\delta_{i, k+l}=-1, k+1 \leq i \leq k+l-1\right),
\end{aligned}
$$

where $E(k, l)$ is of size $(k+l-1) \times(k+l)$.

We will keep this notation throughout this section.

Let $F=\mathbb{Q}(\sqrt{-d}), d \in \mathbb{N}$ square-free, be an imaginary quadratic field. Since we know $r_{4}\left(K_{2} O_{F}\right)$ when $d$ has at most three odd prime divisors (see [18]), we will focus on the cases in which $d$ has at least four odd prime divisors.

First we note that for the symbols generating ${ }_{2} K_{2} O_{F}$, there are some differences between real and imaginary quadratic fields $F$ : there is a nontrivial element (i.e., $\neq 1,2)$ in $F^{*}$ which is in the Tate kernel of an imaginary quadratic field; and we may have $\{-1,-m\} \in \nabla^{2}$ or $\{-1,-m(u+\sqrt{-d})\}$ $\in \nabla^{2}$, where $m$ is a positive divisor of $d$. In particular, it is possible that $\{-1,-1\} \in \nabla^{2}$. In fact, we have

Lemma 4.1. Let $F=\mathbb{Q}(\sqrt{-d}), d \in \mathbb{N}$ square-free, be an imaginary quadratic field. Then $\{-1,-1\} \in \nabla^{2}$ if and only if $d$ or $d / 2$ is of one of the following types: $\left(m_{1}, 0,0,0\right) ;\left(m_{1}, m_{3}, 0,0\right)\left(m_{1} \geq 0\right) ;\left(m_{1}, 0, m_{5}, 0\right)\left(m_{1} \geq 0\right)$.

The proof can be found in [18]. The result will be used repeatedly.

It is not difficult to see that if we take -1 as an element in a system of $\nabla$-representatives, then the remaining elements in the system can be chosen positive, and when $2 \in N F$, the $u$ in the expression $u+\sqrt{-d}$ can be chosen positive. 
As in the real case, we want to find all totally positive rows in any sign matrix (with all possible choices of $\varepsilon_{1}, \ldots, \varepsilon_{k}$, where $k$ is the number of rows of the matrix). Since we know necessary and sufficient conditions for $\{-1,-1\} \in \nabla^{2}$, for simplicity, we shall not consider -1 in any system of $\nabla$-representatives of $F$; instead, we let $\varepsilon \in\{ \pm 1, \pm 2\}$. So except for the case $d \equiv 7(\bmod 8)$, the sign matrices in this section are modified, i.e., we remove -1 from a system of $\nabla$-representatives of $F$.

Theorem 4.1. Let $F=\mathbb{Q}(\sqrt{-d}), d \in \mathbb{N}$ square-free having at least 4 prime divisors, be an imaginary quadratic field.

If $d \equiv 1(\bmod 8)$, then $r_{4}\left(K_{2} O_{F}\right) \geq 1$.

If $d \not \equiv 1(\bmod 8)$ is of type $T=2^{\sigma}\left(m_{1}, m_{3}, m_{5}, m_{7}\right)$, where $\sigma=0$ or 1 , then for any given set $\Lambda=\left\{p_{1}, \ldots, p_{n-1}\right\}$ of odd primes which satisfies $\left(m_{1}, m_{3}, m_{5}, m_{7}\right)$, and for

$P(T, \Lambda)=\left\{p\right.$ prime $\mid d=2^{\sigma} p p_{1} \cdots p_{n-1}$ is of type $\left.T=2^{\sigma}\left(m_{1}, m_{3}, m_{5}, m_{7}\right)\right\}$,

the set

$$
P_{0}(T, \Lambda)=\left\{p \in P(T, \Lambda): r_{4}\left(K_{2} O_{\mathbb{Q}(\sqrt{-d})}\right)=0\right\}
$$

has a positive density. And there exist infinitely many square-free integers $d^{\prime}>0$ of type $T$ such that $r_{4}\left(K_{2} O_{F}\right)>0$, where $F=\mathbb{Q}\left(\sqrt{-d^{\prime}}\right)$.

With some exceptional cases, the same is true for $d$ having at most three odd prime divisors.

Proof. In any case, there is always an element $\tau$ in a system of $\nabla$ representatives such that $\tau \in \nabla^{2}$. If we want to show that $r_{4} \geq 1$, we need to find two such elements. On the other hand, if we show that there is only one such element, then $r_{4}=0$.

The structure of argument here is the same as in the real case. In particular, the treatment of case (iv) there provides a "standard" approach applicable to all cases.

When $T \not \equiv 1(\bmod 8)$, we will give $M_{0}(T)$ and $M_{1}(T)$ in each case. The same approach as in the real case shows that the density of $P_{0}(T, \Lambda)$ is positive. We suggest the following steps to conclude the verification for each case:

\section{STEP 1. Check that $r_{4}=0$ for $M_{0}(T)$.}

STEP 2. Introduce $M(T)$ as in case (iv) of the real case. Choose the values of $x_{j, j}, x_{j, j+1}$ such that $M(T) \cong M_{0}(T)$. If the product of all entries in any row of $M_{0}(T)$ is 1 , the same method as in case (iv) works; if there are some rows in $M_{0}(T)$ for which the product of all entries is -1 , apply elementary row operations to get an equivalent form of $M_{0}(T)$ with the product of all 
entries in the first row equal to -1 , and equal to 1 for the remaining rows. See the illustration of case (vii); if there is a column, e.g., the first one, with all entries 1 in $M_{0}(T)$, apply an elementary operation (III) to $M(T)$ to get all entries 1 in the first column. See the illustration of case (v).

STEP 3. Using Lemma 2.10, show that the density of $P_{0}(T, \Lambda)$ is positive.

On the other hand, Lemma 2.10 implies that there exist infinitely many square-free integers $d \in d(T)$ such that the sign matrices $M(d)$ are the same as $M_{1}(T)$ (in fact, one may choose any prime $p$ satisfying $T$, then choose the remaining primes with described Legendre symbols; clearly, there are infinitely many such $p$ ), which turns out to be that for $F=\mathbb{Q}(\sqrt{d})$, $r_{4}\left(K_{2} O_{F}\right)>0$.

In the following cases $(\mathrm{A})-(\mathrm{H})$ and $(\mathbb{A})-(\mathbb{H})$, for any given type $T$, when we write down $d$, we mean that $d$ is of type $T$. For $i=3,5,7$, we assume that $m_{i} \geq 1(n, s, t \geq 1)$, and only $m_{1}(=m)$ may be 0 . In a sign matrix, we arrange the columns so that the first $t$ columns correspond to $r_{1}, \ldots, r_{t}$, columns $t+1$ to $t+s$ correspond to $q_{1}, \ldots, q_{s}$, columns $t+s+1$ to $t+s+n$ correspond to $p_{1}, \ldots, p_{n}$, and columns $t+s+n+1$ to $t+s+n+m$ correspond to $l_{1}, \ldots, l_{m}$.

\section{(A) $T=\left(m_{1}, 0,0, m_{7}\right)$ :}

A1. $t>0$ even. We have $\left(\frac{u+w}{d}\right)=1$ if $d$ is of type $T$.

Suppose that $M(T)$ is a sign matrix with respect to a given system of $\nabla$-representatives. Applying elementary rows operations to $M(T)$, we find a totally positive row in $M(T)$ since $M(T)$ is of size $(m+t) \times(m+t)$. We assume this row is the last one.

By Lemma 2.9, we know that the product of all entries in any row of $M(T)$ is 1 , hence, if there are two totally positive rows in $M(T)$, then $r_{4} \geq 1$; if this is not the case, then we see that

$$
\begin{gathered}
M(T) \cong\left(\begin{array}{cccc}
-1 & & & -1 \\
& \ddots & & \vdots \\
& & -1 & -1 \\
1 & \ldots & 1 & 1
\end{array}\right) \\
\left(\delta_{i, i}=-1, \delta_{i, m+t}=-1, \text { but } \delta_{m+t, m+t}=1\right) .
\end{gathered}
$$

Multiplying the first $t$ rows together, we get a new row: $\left(-1 \cdots t, 1^{\cdots m}\right)$. Note that the first $t$ columns correspond to $r_{1}, \ldots, r_{t}$. Taking $\varepsilon=-1$ shows that all -1 entries in the row are transformed into 1 . Hence $r_{4} \geq 1$. 
A2. $t$ is odd. Let

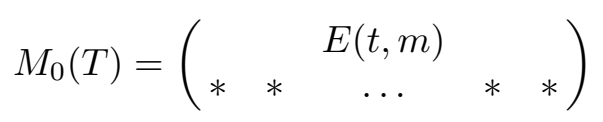

where the last row is arranged to correspond to $u+\sqrt{-d}$, on which we do not put any restriction. For $M_{0}(T)$, one can check that $r_{4}=0$.

To find $d \in d(T)$ with $M(d)=M_{0}(T)$, we choose primes $r_{1}, \ldots, r_{t}$, $l_{1}, \ldots, l_{m}$ and let $\left\{-r_{1}, \ldots,-r_{t}, l_{1}, \ldots, l_{m-1}, u+\sqrt{-d}\right\}$ if $m>0$, and $\left\{-r_{1}, \ldots,-r_{t-1}, u+\sqrt{-d}\right\}$ if $m=0$, be a system of $\nabla$-representatives.

If $t \geq 3$, we put

$$
M_{1}(T)=\left(\begin{array}{ccccc}
1 & 1 & 1 & \ldots & 1 \\
-1 & -1 & 1 & \ldots & 1 \\
-1 & -1 & 1 & \ldots & 1 \\
\vdots & \vdots & \vdots & & \vdots
\end{array}\right)
$$

If $m \geq 2$, we put

$$
M_{1}(T)=\left(\begin{array}{ccccc}
1 & 1 & 1 & \ldots & 1 \\
* & * & * & \ldots & * \\
\vdots & \vdots & \vdots & & \vdots \\
* & * & * & \ldots & * \\
1 & 1 & 1 & \ldots & 1
\end{array}\right)
$$

where the first and last rows are totally positive. Then we have $r_{4} \geq 1$. Note that if $m=1$, then $t \geq 3$.

As in the real quadratic case, without loss of generality, we will assume $m>0$ when we give a system of $\nabla$-representatives and sign matrices in the following.

(B) $T=\left(m_{1}, 0, m_{5}, 0\right)$ : We have $\{-1,-1\} \in \nabla^{2}$.

B1. $s>0$ even. Hence $T \equiv 1(\bmod 8)$. For $M(T)=D(s+m)$, we have the minimum $r_{4}$. Multiplying together the first $s-1$ rows if $m=0$, or the first $s$ rows if $m \geq 1$, we get a row vector which has -1 in the first $s$ entries and 1 elsewhere. Since $\left(\frac{2}{p}\right)=-1$ for any prime $p \equiv 5(\bmod 8)$, taking $\varepsilon=2$, we obtain another element in $\nabla^{2}$. Therefore $r_{4} \geq 1$.

B2. $s$ is odd. Let $M_{0}(T)=D(s+m)$. Then $r_{4}=0$.

To find $d \in d(T)$ with $M(d)=M_{0}(T)$, we choose primes $q_{1}, \ldots, q_{s}$, $l_{1}, \ldots, l_{m}$ and let $\left\{q_{1}, \ldots, q_{s}, l_{1}, \ldots, l_{m-1}\right\}$ be a system of $\nabla$-representatives.

On the other hand, taking the first row of $M_{1}(T)$ totally positive, we see that $r_{4} \geq 1$. 
(C) $T=\left(m_{1}, m_{3}, 0,0\right)$ : We have $\{-1,-1\} \in \nabla^{2}$.

C1. $n>0$ is even. Since $\left(\frac{2}{p}\right)=-1$ for any prime $p \equiv 3(\bmod 8)$, the situation here is the same as the case of $\left(m_{1}, 0, m_{5}, 0\right)$ with $s=m_{5}>0$ even.

C2. $n$ is odd. Let $M_{0}(T)=E(n, m)$. Then $r_{4}=0$.

To find $d \in d(T)$ with $M(d)=M_{0}(T)$, we choose primes $p_{1}, \ldots, p_{n}$, $l_{1}, \ldots, l_{m}$ and let $\left\{-p_{1}, \ldots,-p_{n}, l_{1}, \ldots, l_{m-1}\right\}$ be a system of $\nabla$-representatives. For this system, Lemma 2.3 shows that we can choose $\eta=1$ always.

If we take the first row of $M_{1}(T)$ totally positive, then $r_{4}>0$.

(D) $T=\left(m_{1}, 0, m_{5}, m_{7}\right)$ :

D1. $T \equiv 1(\bmod 8)$, i.e., both $t$ and $s$ are even (positive). For $M(T)=$ $D(t+s+m)$, we have the minimum $r_{4}$. Multiplying the first $t$ rows together and taking $\varepsilon=-1$ and multiplying rows $t+1$ to $t+s$ together and taking $\varepsilon=2$, respectively, we get two distinct elements in $\nabla^{2}$. Hence, $r_{4} \geq 1$.

D2. $T \not \equiv 1(\bmod 8)$. Let $M_{0}(T)=E(t, s+m)$. Then $r_{4}=0$. In fact, one can get the unique element in $\nabla^{2}$ by

- taking $\varepsilon=-1$ to the $t$ th row, if $t$ is even and $s$ is odd;

- multiplying rows $t+1$ to $t+s$ together and taking $\varepsilon=2$, if $s$ is even and $t$ is odd;

- multiplying rows $t$ to $t+s$ and taking $\varepsilon=-2$, if both $s$ and $t$ are odd.

Note that if both $t$ and $s$ are even, then $T \equiv 1(\bmod 8)$, which is the case of D1.

To find $d \in d(T)$ with $M(d)=M_{0}(T)$, we choose primes $r_{1}, \ldots, r_{t}$, $q_{1}, \ldots, q_{s}, l_{1}, \ldots, l_{m}$ and let $\left\{-r_{1}, \ldots,-r_{t}, q_{1}, \ldots, q_{s}, l_{1}, \ldots, l_{m-1}\right\}$ be a system of $\nabla$-representatives.

We can take the first row of $M_{1}(T)$ totally positive in any case and take the $(t+1)$ th row totally positive if $s+m \geq 2$, or the second as $(-1, \ldots,-1,1)$ if $s=1, m=0$ and $t$ is even, or the second totally negative if $s=1, m=0$ and $t$ is odd. Then $r_{4}>0$.

(E) $T=\left(m_{1}, m_{3}, 0, m_{7}\right)$ :

E1. $T \equiv 1(\bmod 8)$ is the same as D1.

E2. $T \not \equiv 1(\bmod 8)$. Let $M_{0}(T)=E(t+n, m)$. Then $r_{4}=0$ and the unique element in $\nabla^{2}$ can be obtained by

- taking $\varepsilon=-1$ to the $(t+n)$ th row, if $t+n$ is even (hence both $t$ and $n$ are odd);

- taking $\varepsilon=-2$ to the $t$ th row, if $t$ is even and $n$ is odd; 
- multiplying the $t$ th and the $(t+n)$ th rows together and taking $\varepsilon=2$, if $t$ is odd and $n$ is even.

To find $d \in d(T)$ with $M(d)=M_{0}(T)$, we choose primes $r_{1}, \ldots, r_{t}$, $p_{1}, \ldots, p_{n}, l_{1}, \ldots, l_{m}$ and let $\left\{-r_{1}, \ldots,-r_{t},-p_{1}, \ldots,-p_{n}, l_{1}, \ldots, l_{m-1}\right\}$ be a system of $\nabla$-representatives.

To obtain $r_{4}>0$, we may take the first row of $M_{1}(T)$ totally positive and both the second and third to be $(-1,-1,1, \ldots, 1)$.

(F) $T=\left(m_{1}, m_{3}, m_{5}, 0\right)$ :

F1. $T \equiv 1(\bmod 8)$ is the same as D1.

F2. $T \not \equiv 1(\bmod 8)$. Let

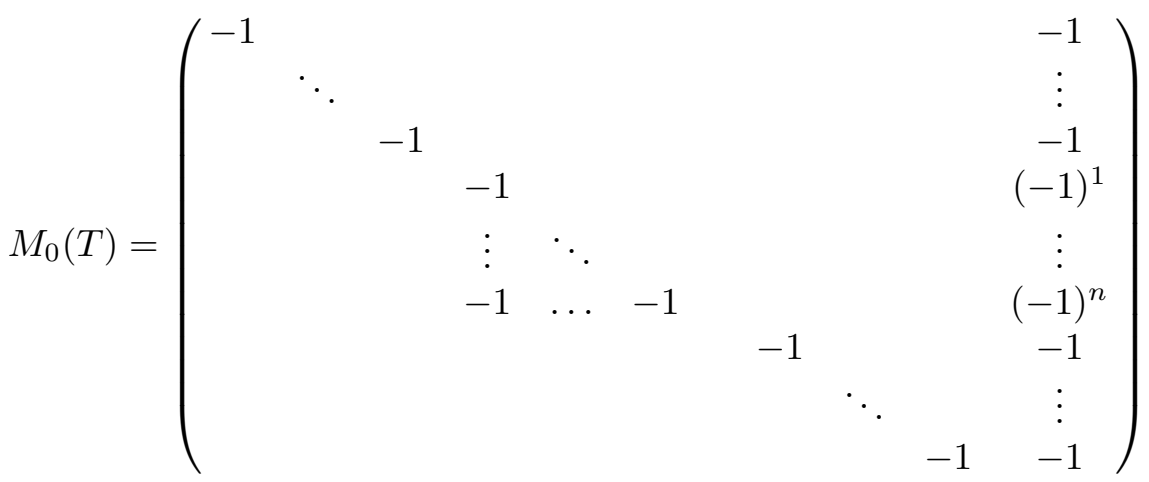

$$
\begin{aligned}
& \begin{array}{c}
\left(\delta_{i, i}=\delta_{i, s+n+m}=-1,1 \leq i \leq s ;\right. \\
\delta_{i, j}=-1, \delta_{i, s+n+m}=(-1)^{i-s}, s+1 \leq i \leq s+n \\
\left.\delta_{i, i}=\delta_{i, s+n+m}=-1, s+n+1 \leq i \leq s+n+m-1\right) .
\end{array}
\end{aligned}
$$

Then one can check as above that $r_{4}=0$.

To find $d \in d(T)$ with $M(d)=M_{0}(T)$, we choose primes $q_{1}, \ldots, q_{s}$, $p_{1}, \ldots, p_{n}, l_{1}, \ldots, l_{m}$ and let $\left\{q_{1}, \ldots, q_{s},-p_{1}, \ldots,-p_{n}, l_{1}, \ldots, l_{m-1}\right\}$ be a system of $\nabla$-representatives.

If we take the first two rows of $M_{1}(T)$ totally positive, then we get $r_{4}>0$.

(G) $T=\left(m_{1}, m_{3}, m_{5}, m_{7}\right)$ :

G1. $T \equiv 1(\bmod 8)$. We must have $t \equiv s \equiv n(\bmod 2)$.

For $M(T)=D(t+s+n)$, we have the minimum $r_{4}$. Multiplying the first $t+s$ rows together and taking $\varepsilon=-2$, and multiplying rows from $t+1$ to $t+s+n$ if $m>0$, and to $t+s+n-1$ if $m=0$, and taking $\varepsilon=2$, respectively, we will obtain two elements in $\nabla^{2}$. 
G2. $T \not \equiv 1(\bmod 8)$. Let

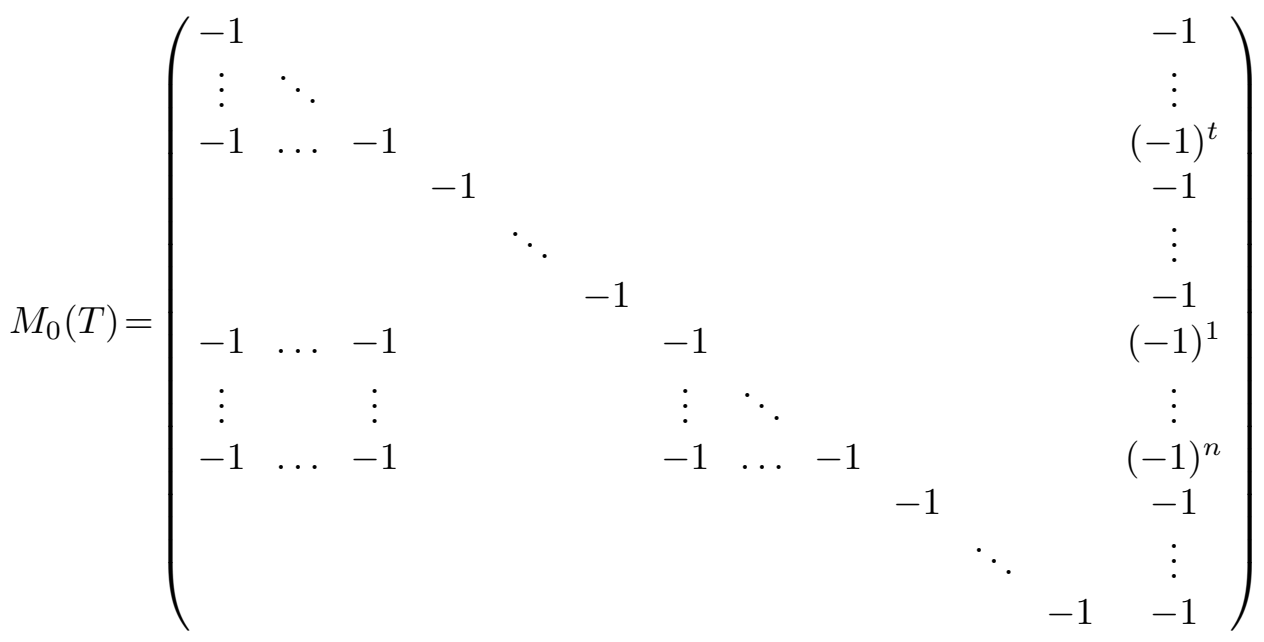

$$
\begin{aligned}
& \left(\delta_{i, j}=-1, \delta_{i, t+s+n+m}=(-1)^{i}, 1 \leq j \leq i \leq t\right. \\
& \delta_{i, i}=\delta_{i, t+s+n+m}=-1, t+1 \leq i \leq s+t \text {; } \\
& \delta_{i, j}=-1, \delta_{i, t+s+n+m}=(-1)^{i-(t+s)}, s+t+1 \leq i \leq s+t+n, 1 \leq j \leq t \\
& \text { or } s+t+1 \leq j \leq i \\
& \left.\delta_{i, i}=\delta_{i, t+s+n+m}=-1, s+t+n+1 \leq i \leq s+t+n+m-1\right) .
\end{aligned}
$$

Then we have $r_{4}=0$.

To find $d \in d(T)$ with $M(d)=M_{0}(T)$, we choose primes $r_{1}, \ldots, r_{t}$, $q_{1}, \ldots, q_{s}, p_{1}, \ldots, p_{n}, l_{1}, \ldots, l_{m}$ and let $\left\{-r_{1}, \ldots,-r_{t}, q_{1}, \ldots, q_{s},-p_{1}, \ldots\right.$ $\left.\ldots,-p_{n}, l_{1}, \ldots, l_{m-1}\right\}$ be a system of $\nabla$-representatives.

To obtain $r_{4}>0$, we can take the first row and the $(t+1)$ th of $M_{1}(T)$ totally positive.

(H) $T=\left(m_{1}, 0,0,0\right)$ : In this case, $\{-1,-1\} \in \nabla^{2}$.

We have $\left(\frac{u+w}{d}\right)=1$. Since any sign matrix is of size $m \times m$, there exists another element $\in \nabla^{2}$. Hence $r_{4} \geq 1$.

We now turn to the case in which $d \in d(T)$ is even. We will give $M_{0}(T)$ and $M_{1}(T)$.

To find $d \in d(T)$ with $M(d)=M_{0}(T)$, we choose primes $r_{1}, \ldots, r_{t}$, $q_{1}, \ldots, q_{s}, p_{1}, \ldots, p_{n}, l_{1}, \ldots, l_{m}$ such that $d=2 \cdot r_{1} \cdots r_{t} \cdot q_{1} \cdots q_{s} \cdot p_{1} \cdots p_{n}$. $l_{1} \cdots l_{m}$ is of type $T$. Here $t, s, n$ and $m$ are allowed to be 0 . We choose $\left\{r_{1}, \ldots, r_{t}, l_{1}, \ldots, l_{m-1}, u+\sqrt{-d}\right\}$ and $\left\{l_{1}, \ldots, l_{m-1}, u+\sqrt{-d}\right\}$ as a system of $\nabla$-representatives for cases $(\mathbb{A})$ and $(\mathbb{H})$ respectively, and we choose $\left\{r_{1}, \ldots, r_{t}, q_{1}, \ldots, q_{s}, p_{1}, \ldots, p_{n}, l_{1}, \ldots, l_{m-1}\right\}$ for the other cases. Without loss of generality, we assume that $m>0$ in the following. 
(A) $T=2\left(m_{1}, 0,0, m_{7}\right)$ :

A1. $t>0$ even. We choose $\left\{r_{1}, \ldots, r_{t}, l_{1}, \ldots, l_{m-1}, u+\sqrt{-d}\right\}$ as a system of $\nabla$-representatives. Let

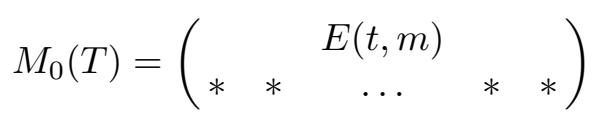

where the last row is arranged to correspond to $u+\sqrt{-d}$. We observe that a necessary condition for $(u+w) m Z^{2}=X^{2}-d Y^{2}$ to have a non-trivial solution is $\left(\frac{u+w}{d / 2}\right)=1$, where $m$ is a divisor of $d$. It follows that if the product of all elements in the last row is -1 , then $r_{4}=0$. We see that the product is 1 if and only if $\left(\frac{2}{|u+w|}\right)=1$ if and only if $d / 2 \equiv 1(\bmod 16)$. So letting the sign matrix be as above and letting $d_{1} \equiv 9(\bmod 16)$, we have $M_{0}(T)$.

To obtain $r_{4}>0$, we can take the first two rows of $M(T)$ to be $(1, \ldots, 1)$ and $\left(-1^{\cdots t}, 1^{\cdots m}\right)$.

A2. $t$ odd. Let

$$
\begin{aligned}
& M_{0}(T)=\left(\begin{array}{cccccccc}
1 & 1 & 1 & \ldots & & & & -1 \\
-1 & 1 & 1 & \ldots & & & & 1 \\
-1 & -1 & 1 & \ldots & & & & -1 \\
\vdots & \vdots & \vdots & \ddots & & & & \vdots \\
-1 & -1 & -1 & \ldots & 1 & & & -1 \\
& & & & & -1 & & -1 \\
& & & & & & \ddots & \\
& & & & & & -1 & -1 \\
* & * & * & \ldots & & & * & *
\end{array}\right) \\
& \left(\delta_{i, j}=-1, \delta_{k, t+m}=(-1)^{k}, 1 \leq j<i \leq t, 1 \leq k \leq t ;\right. \\
& \left.\delta_{i, i}=\delta_{i, t+m}=-1, t+1 \leq i \leq t+m-1\right),
\end{aligned}
$$

where the last row is arranged to correspond to $u+\sqrt{-d}$. Then we have $r_{4}=0$.

To obtain $r_{4}>0$, we can take the first two rows of $M(T)$ to be

$$
\left(-1^{\cdots t}, 1^{\cdots m}\right) \text { and }(1, \ldots, 1) \text {. }
$$

(B) $T=2\left(m_{1}, 0, m_{5}, 0\right)$ : We have $\{-1,-1\} \in \nabla^{2}$.

For the sign matrix 


$$
\begin{aligned}
& M_{0}(T)=\left(\begin{array}{ccccccc}
1 & & & & & & \\
& -1 & & & & & 1 \\
& & \ddots & & & & \vdots \\
& & & -1 & & & 1 \\
& & & -1 & & -1 \\
& & & & \ddots & & \vdots \\
& & & & & -1 & -1
\end{array}\right) \\
& \left(\delta_{i, i}=-1,2 \leq i \leq s, \delta_{1, s+m}=-1 ;\right. \\
& \left.\delta_{i, i}=\delta_{i, s+m}=-1, s+1 \leq i \leq s+m-1\right) \text {, }
\end{aligned}
$$

we have $r_{4}=0$, while for

$$
\begin{aligned}
& M(T)=\left(\begin{array}{ccccccc}
1 & & & & & & -1 \\
& \ddots & & & & \\
& & 1 & & & -1 \\
& & & 1 & \ldots & 1 \\
& & & & \ddots & & \vdots \\
& & & & & 1 & 1
\end{array}\right) \\
& \left(\delta_{i, s+m}=-1,1 \leq i \leq s\right),
\end{aligned}
$$

we have $r_{4} \geq 1$.

(C) $T=2\left(m_{1}, m_{3}, 0,0\right)$ : We have $\{-1,-1\} \in \nabla^{2}$.

Let

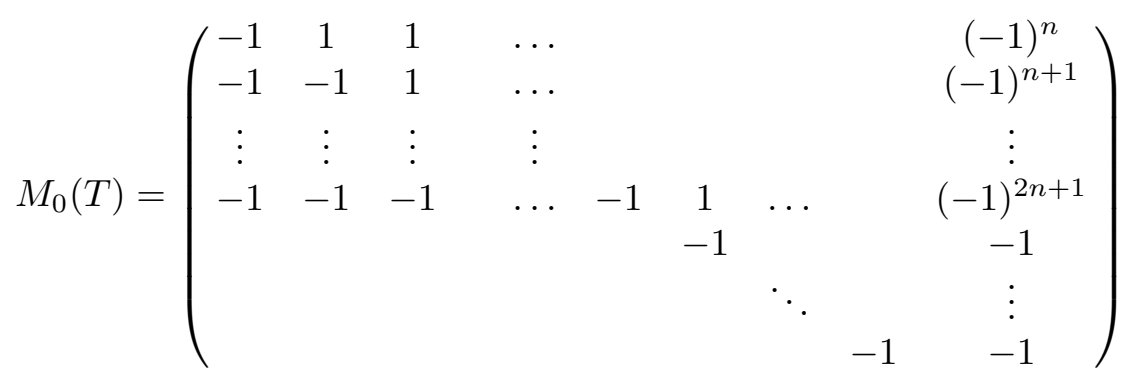

$$
\begin{aligned}
& \left(\delta_{i, j}=-1, \delta_{i, n+m}=(-1)^{n+i-1}, 1 \leq j \leq i \leq n ;\right. \\
& \left.\delta_{i, i}=\delta_{i, n+m}=-1, n+1 \leq i \leq n+m-1\right) .
\end{aligned}
$$

Then we have $r_{4}=0$.

On the other hand, if we let the first two rows of $M(T)$ be $(-1,1, \ldots, 1)$ if $n$ is even, and let the first row be totally positive if $n$ is odd, then $r_{4} \geq 1$. 
(D) $T=2\left(m_{1}, 0, m_{5}, m_{7}\right):$ Let

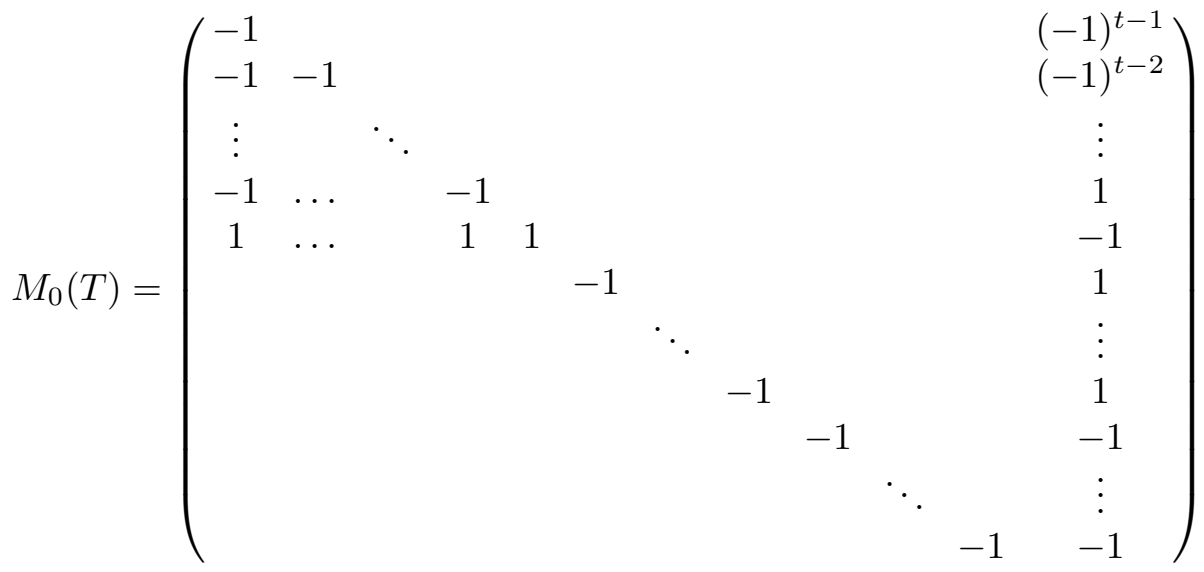

$$
\begin{aligned}
& \left(\delta_{i, j}=-1, \delta_{i, t+s+m}=(-1)^{t-i}, 1 \leq j \leq i \leq t ;\right. \\
& \delta_{t+1, t+s+m}=-1, \delta_{i, i}=-1, t+2 \leq i \leq t+s \\
& \left.\delta_{i, i}=\delta_{i, t+s+m}=-1, t+s+1 \leq i \leq t+s+m-1\right) \text {. }
\end{aligned}
$$

Then $r_{4}=0$.

To obtain $r_{4}>0$, if $t(>0)$ is even, we can take the first two rows to be $(1, \ldots, 1)$ and $\left(-1^{\cdots t}, 1^{\cdots s+m}\right)$; if both $s$ and $t$ are odd, we can take them to be $\left(-1^{\cdots t}, 1^{\cdots s+m}\right)$ and $\left(1^{\cdots t},-1^{\cdots s}, 1^{\cdots m}\right)$; if $s$ is even, take the first row to be $\left(-1 \cdots t, 1^{\cdots s+m}\right)$ and the $(t+1)$ th and $(t+2)$ th to be $(1, \ldots, 1,-1)$.

(E) $T=2\left(m_{1}, m_{3}, 0, m_{7}\right):$ Let

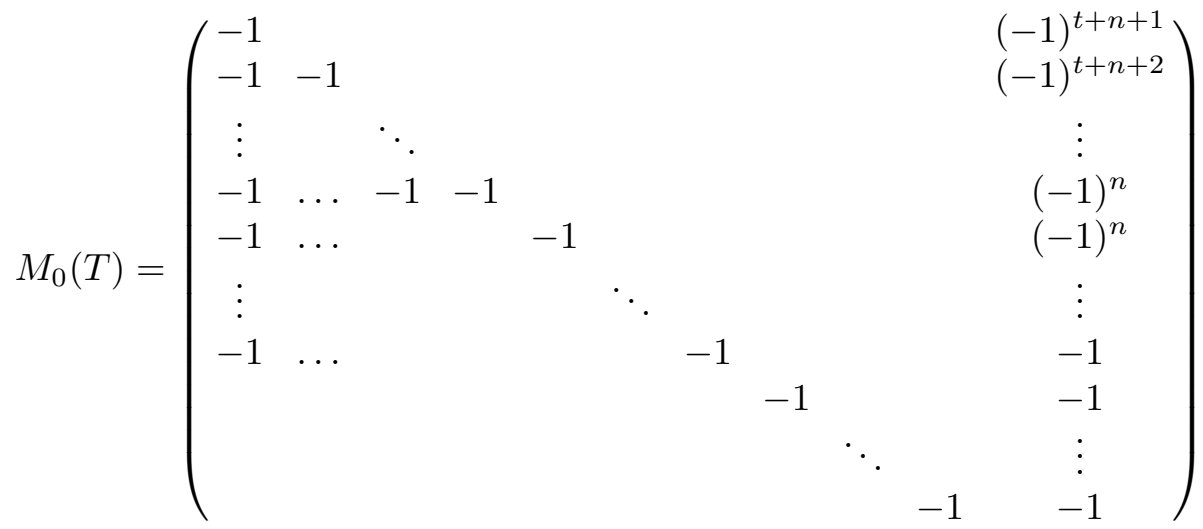

$$
\begin{aligned}
& \left(\delta_{i, j}=-1, \delta_{i, t+n+m}=(-1)^{i+t+n}, 1 \leq j \leq i \leq t ;\right. \\
& \delta_{i, j}=-1, \delta_{i, t+n+m}=(-1)^{t+n+1-i}, t+1 \leq j \leq i \leq t+n \text {; } \\
& \left.\delta_{i, i}=\delta_{i, t+n+m}=-1, t+n+1 \leq i \leq t+n+m-1\right) .
\end{aligned}
$$

Then $r_{4}=0$. 
To obtain $r_{4}>0$, when $t+n$ is even, if $t \geq 2$, we can take the first row totally positive and the second $\left(-1^{\cdots t+n}, 1^{\cdots m}\right)$; if $t=1$, take the first row totally positive and the second $(-1,1, \ldots, 1)$; when $t+n$ is odd, we can take the first row to be $\left(-1^{\cdots t+n}, 1^{\cdots m}\right)$ and the $(t+1)$ th totally positive.

(F) $T=2\left(m_{1}, m_{3}, m_{5}, 0\right):$ Let

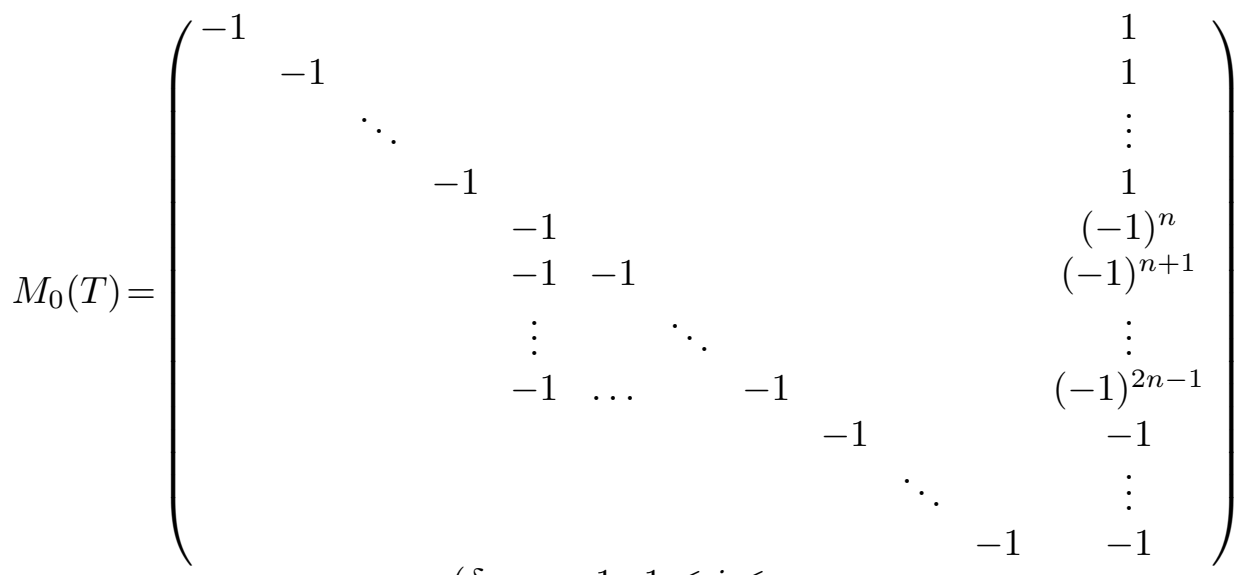

$$
\begin{aligned}
& \left(\delta_{i, i}=-1,1 \leq i \leq s ;\right. \\
& \delta_{i, j}=-1, \delta_{i, s+n+m}=(-1)^{i-s+n-1}, s+1 \leq j \leq i \leq s+n ; \\
& \left.\delta_{i, i}=\delta_{i, s+n+m}=-1, s+n+1 \leq i \leq s+n+m-1\right) .
\end{aligned}
$$

Then $r_{4}=0$.

To obtain $r_{4}>0$, we first assume that $n(>0)$ is even. If $s$ is also even, we can take the first three rows to be $(1, \ldots, 1,-1)$; if $s$ is odd, take the first two rows to be $\left(-1^{\cdots s+n}, 1^{\cdots m}\right)$. Assume now $n$ is odd. If $s \geq 2$, we can take the first two rows to be $(1 \cdots s,-1 \cdots n, 1 \cdots m)$; if $s=1$, take the first row to be $\left(1,-1^{\cdots n}, 1^{\cdots m}\right)$ and the second $\left(-1^{\cdots 1+n}, 1^{\cdots m}\right)$.

(G) $T=2\left(m_{1}, m_{3}, m_{5}, m_{7}\right)$ : Let $M_{0}(T)$ be

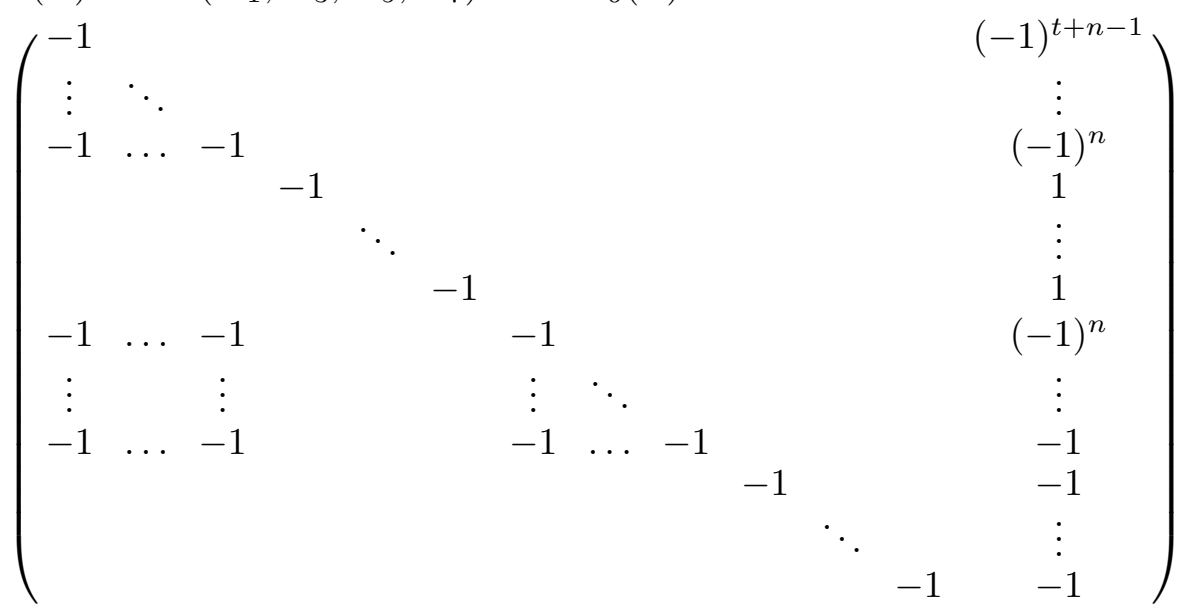




$$
\begin{gathered}
\left(\delta_{i, j}=-1, \delta_{i, t+s+n+m}=(-1)^{t+n-i}, 1 \leq j \leq i \leq t ;\right. \\
\delta_{i, i}=-1, t+1 \leq i \leq t+s ; \\
\delta_{i, j}=-1, \delta_{i, t+s+n+m}=(-1)^{t+s+n+1-i}, t+s+1 \leq i \leq t+s+n, 1 \leq j \leq t \\
\quad \text { or } s+t+1 \leq j \leq i ; \\
\left.\delta_{i, i}=\delta_{i, t+s+n+m}=-1, t+s+n+1 \leq i \leq t+s+n+m-1\right) .
\end{gathered}
$$

To obtain $r_{4}>0$, we need to consider two cases.

$\mathbb{G} 1$. $t+n$ is even: if $t \geq 2$, take the first row totally positive and the second $\left(-1^{\cdots t}, 1^{\cdots s},-1^{\cdots n}, 1^{\cdots m}\right)$; if $t=1$ and $s \geq 2$, take the first row totally positive and the second and third to be $(1, \ldots, 1,-1)$; if $t=s=1, n \geq 3$, we can take the first three rows to be $(-1,1,-1 \cdots n, 1 \cdots m),(1,-1,1, \ldots, 1)$ and $\left(1,1,-1^{\cdots n}, 1^{\cdots m}\right)$; if $t=s=n=1$, then $m \geq 1$, take the first row totally positive and the second and third to be $(1,-1,1, \ldots, 1)$ and $(-1,1, \ldots, 1)$.

$\mathbb{G} 2$. $t+n$ is odd: if $n+m \geq 2$, take the first row to be

$$
\left(-1^{\cdots t}, 1^{\cdots s},-1^{\cdots n}, 1^{\cdots m}\right)
$$

and the $(t+s+1)$ th row totally positive; if $n=1, m=0$, take the first row to be $(-1 \cdots t, 1 \cdots s,-1)$ and the second and $(t+1)$ th to be $\left(1^{\cdots t+s},-1\right)$.

$(\mathbb{H}) T=2\left(m_{1}, 0,0,0\right)$ :

Let

$$
\begin{gathered}
M(T)=\left(\begin{array}{cccc}
-1 & & & -1 \\
& \ddots & & \vdots \\
& & -1 & -1 \\
* & \ldots & * & *
\end{array}\right) \\
\left(\delta_{i, i}=\delta_{i, m}=-1,1 \leq i \leq m-1\right) .
\end{gathered}
$$

Here the last row corresponds to $u+\sqrt{-d}$. Then $r_{4}=0$ if and only if the product of the entries in the last row is -1 . Again, as in the case of $2\left(m_{1}, 0,0, m_{7}\right)$ with $t=m_{7}$ even, we see that this product is 1 if and only if $\left(\frac{2}{|u+w|}\right)=1$ if and only if $d_{1} \equiv 1(\bmod 16)$.

So letting the sign matrix as above and letting $d / 2 \equiv 9(\bmod 16)$, we have $M_{0}(T)$.

And if we take the first $m-1$ rows totally positive, then we see that $r_{4} \geq 1$.

This completes the proof.

COROLlary 4.1. In cases $(\mathbb{A})-(\mathbb{H})$ and in subcases 2 of $(\mathrm{A})-(\mathrm{G})$, if we choose a system of $\nabla$-representatives as in the proof, then $\left(\mathrm{K}_{2} \mathrm{O}_{\mathrm{F}}\right)_{2}$ is elementary abelian if and only if $M(d) \cong M_{0}(T)$. 
Corollary 4.2. Let $F=\mathbb{Q}(\sqrt{-2 d})$ with $d \equiv 1(\bmod 16)$ of type $\left(m_{1}, 0,0,0\right)$. Then $r_{4}\left(K_{2} O_{F}\right) \geq 1$.

Suppose that $d \in \mathbb{N}$ is square-free and has $n$ prime divisors. We shall give some examples to show why in the imaginary case we have no analogue of the statement of Theorem 3.1 for $r_{4}\left(K_{2} O_{F}\right)>0$.

EXAMPLE 4.1. Let $d=l_{1} \cdots l_{m} \cdot p$, where $l_{i} \equiv 1(\bmod 8)$ and $p \equiv 3$ or 5 $(\bmod 8)$ are primes. We choose $\left\{l_{1}, \ldots, l_{m}\right\}$ as a system of $\nabla$-representatives and let the sign matrix $M(d)$ be

$$
\begin{gathered}
M(d)=\left(\begin{array}{cccccc}
* & -1 & 1 & \ldots & 1 & * \\
1 & * & -1 & \ldots & 1 & * \\
1 & 1 & * & \ldots & 1 & * \\
\vdots & \vdots & \vdots & \ddots & \vdots & \vdots \\
1 & 1 & 1 & \ldots & -1 & * \\
-1 & 1 & 1 & \cdots & * & *
\end{array}\right) \\
\left(\delta_{i, i+1}=-1, \delta_{m, 1}=-1,1 \leq i \leq m\right) .
\end{gathered}
$$

Here all $*$ depend on the choice of $p$ and we have arranged the first $m$ columns according to $l_{1}, \ldots, l_{m}$. One sees that for any choice of prime $p, r_{4}\left(K_{2} O_{F}\right)$ $=0$, where $F=\mathbb{Q}(\sqrt{d})$. One can also see that with the same sign matrix, for $p \equiv 7(\bmod 8), r_{4}\left(K_{2} O_{F}\right)=0$ also holds. Moreover, the same is true for $d=2 l_{1} \cdots l_{m} \cdot p$ if $p \not \equiv 1(\bmod 8)$.

REmarks. 1. One sees that our proof (hence the assertion) is also valid for the case in which $d$ has three odd prime divisors except the case of $d=2 l q p$, where $l, q, p \equiv 7,5,3(\bmod 8)$ are primes, in which we always have $r_{4}=0$. See [18].

2. By the results in [18], we have the statements of Theorem 4.1 when $d$ has two odd prime divisors if and only if $d=2 d_{1}$, or $d_{1} d_{3}$, or $2 d_{1} d_{3}$, or $2 d_{1} d_{5}$, or $d_{1} d_{5}$, or $d_{1} d_{7}$, or $2 d_{1} d_{7}$, or $2 d_{7}$. Here $d_{1} \neq 1$.

3. By Theorem 4.1 and by [18] again, we see that if $d \equiv 1(\bmod 8)$, then $r_{4}>0$ for all $d \neq 1$.

Acknowledgments. The author would like to thank the Abdus Salam International Centre for Theoretical Physics, Trieste, Italy, and Centre for Theoretical Science in Taiwan, for their hospitality. He is in great debt to Professor M. Kolster for his suggestions and reformulating some results of this paper. He is also grateful to J. Browkin, J. Hurrelbrink, F. Keune, A. O. Kuku and R. Osburn for their valuable help. He wishes particularly to thank the referee for his/her careful reading, valuable comments and suggestions. 


\section{References}

[1] B. Brauckmann, The 2-Sylow subgroups of the tame kernel of number fields, Canad. J. Math. 43 (1991), 255-264.

[2] J. Browkin and H. Gangl, Table of tame and wild kernels of quadratic imaginary number fields, Math. Comp. 68 (1999), 291-305.

[3] J. Browkin and A. Schinzel, On 2-Sylow subgroups of $\mathrm{K}_{2} \mathrm{O}_{F}$ for quadratic fields, J. Reine Angew. Math. 331 (1982), 104-113.

[4] A. Candiotti and K. Kramer, On the 2-Sylow subgroup of the Hilbert kernel of $K_{2}$ of number fields, Acta Arith. 52 (1989), 49-65.

[5] P. E. Conner and J. Hurrelbrink, Class Number Parity, World Sci., Singapore, 1988.

[6] - , 一, The 4-rank of $K_{2}(o)$, Canad. J. Math. 41 (1989), 932-960.

[7] - - - On elementary abelian 2-Sylow $K_{2}$ of rings of integers of certain quadratic number fields, Acta Arith. 73 (1995), 59-65.

[8] J. Hurrelbrink and M. Kolster, Tame kernels under relative quadratic extensions and Hilbert symbol, J. Reine Angew. Math. 499 (1998), 145-188.

[9] K. Ireland and M. Rosen, A Classical Introduction to Modern Number Theory, Springer, New York, 1982.

[10] F. Keune, On the structure of the $K_{2}$ of the ring of integers in a number field, K-Theory 2 (1989), 625-645.

[11] M. Kolster, The structure of the 2-Sylow subgroup of $K_{2}(O) I$, Comment. Math. Helv. 61 (1986), 376-388.

[12] B. Mazur and A. Wiles, Class fields of abelian extensions of $\mathbb{Q}$, Invent. Math. 76 (1984), 179-330.

[13] L. J. Mordell, Diophantine Equations, Academic Press, London, New York, 1969.

[14] J. Neukirch, Class Field Theory, Springer, Berlin, 1986.

[15] O. T. O'Meara, Introduction to Quadratic Forms, Springer, Berlin, 1963.

[16] R. Osburn, Densities of 4-ranks of $K_{2}(O)$, to appear.

[17] H. R. Qin, 2-Sylow subgroups of $K_{2} O_{F}$ for real quadratic fields $F$, Sci. in China 37 (1994), 1302-1313.

[18] - The 2-Sylow subgroups of the tame kernel of imaginary quadratic fields, Acta Arith. 69 (1995), 153-169.

[19] - , The 4-rank of $\mathrm{K}_{2} \mathrm{O}_{F}$ for real quadratic fields, ibid. 72 (1995), 323-333.

[20] - , Tame kernels and Tate kernels of quadratic number fields, J. Reine Angew. Math. 530 (2001), 105-144.

[21] J. Rognes and C. Weibel (with an appendix by M. Kolster), Two-primary algebraic K-theory of rings of integers in number fields, J. Amer. Math. Soc. 13 (2000), 1-54.

[22] A. A. Suslin, Torsion in $K_{2}$ of fields, K-Theory 1 (1987), 5-29.

[23] J. Tate, Relations between $K_{2}$ and Galois cohomology, Invent. Math. 36 (1976), $257-274$.

[24] J. Urbanowicz, On the 2-primary part of a conjecture of Birch and Tate, Acta Arith. 43 (1983), 69-81.

[25] A. Vazzana, On the 2-primary part of $K_{2}$ of rings of integers in certain quadratic number fields, ibid. 80 (1997), 225-235.

[26] A. Wiles, The Iwasawa conjecture for totally real fields, Ann. of Math. 131 (1990), 493-540.

[27] X. B. Yin and H. R. Qin, The structure of the tame kernels of quadratic number fields $(I I)$, to appear. 
[28] X. B. Yin and H. R. Qin, The structure of the tame kernels of quadratic number fields $(I I I)$, to appear.

Department of Mathematics

Nanjing University

Nanjing 210093, P.R. China

E-mail: hrqin@nju.edu.cn

Received on 15.4.2002

and in revised form on 4.8.2003 